\title{
Dorado and its member galaxies $\mathrm{H} \alpha$ imaging of the group backbone ${ }^{\star}$
}

\author{
R. Rampazzo ${ }^{1,2}$, S. Ciroi ${ }^{3}$, P. Mazzei ${ }^{2}$, F. Di Mille ${ }^{4}$, E. Congiu ${ }^{4,5}$, A. Cattapan ${ }^{3}$, L. Bianchi ${ }^{6}$, E. Iodice ${ }^{7}$, A. Marino ${ }^{2}$, \\ H. Plana ${ }^{8}$, J. Postma ${ }^{9}$, and M. Spavone ${ }^{7}$
}

1 INAF-Osservatorio Astrofisico di Asiago, Via dell'Osservatorio 8, 36012 Asiago, Italy e-mail: roberto.rampazzo@inaf.it

2 INAF-Osservatorio Astronomico di Padova, Vicolo dell'Osservatorio 5, 35122 Padova, Italy

3 Department of Physics and Astronomy “G. Galilei”, University of Padova, Vicolo dell'Osservatorio 3, 35122 Padova, Italy

${ }^{4}$ Las Campanas Observatory, Carnegie Institution of Washington, Colina El Pino Casilla 601, La Serena, Chile

5 Departamento de Astronomía, Universidad de Chile, Camino del Observatorio 1515, Las Condes, Santiago, Chile

6 Dept. of Physics \& Astronomy, The Johns Hopkins University, 3400 N. Charles St., Baltimore, MD 21218, USA

7 INAF-Osservatorio Astronomico di Capodimonte, Salita Moiariello 16, 80131 Napoli, Italy

${ }^{8}$ Laboratório de Astrofísica Teórica e Observacional, Universidade Estadual de Santa Cruz, 45650-000 Ilhéus, BA, Brasil

9 University of Calgary, 2500 University, Drive NW, Calgary, Alberta, Canada

Received 22 July 2020 / Accepted 29 September 2020

\section{ABSTRACT}

Context. Dorado is a nearby, rich and clumpy galaxy group that extends for several degrees in the southern hemisphere. Although several studies have been dedicated to defining its members, their kinematics, and the hot and cold gas content, in particular $\mathrm{HI}$, their present star formation activity remains unknown.

Aims. For the first time, we map the $\mathrm{H} \alpha$ distribution as a possible indicator of the star formation activity of Dorado members, a large fraction of which show interaction and merging signatures independently of their morphological type.

Methods. With the $2.5 \mathrm{~m}$ du Pont and the $1 \mathrm{~m}$ Swope telescopes, we obtained narrow-band calibrated images of 14 galaxies that form the backbone of the group, mapping $\mathrm{H} \alpha+\left[\mathrm{N}\right.$ II] down to a few $10^{-17} \mathrm{erg} \mathrm{cm}^{-2} \mathrm{~s}^{-1} \operatorname{arcsec}^{-2}$. We estimated the galaxy star formation rate from the $\mathrm{H} \alpha$ fluxes and corrected for Galaxy foreground extinction and [N II] contamination.

Results. We detected $\mathrm{H} \alpha+[\mathrm{N} \mathrm{II}]$ emission in all galaxies. H II regions clearly emerge in late-type galaxies, while in early-type galaxies the $\mathrm{H} \alpha+[\mathrm{N} \mathrm{II}]$ emission is dominated by [N II], especially in the central regions. However, H II complexes are revealed in four earlytype galaxies. Even in the compact group SGC 0414-5559, in the projected centre of Dorado, H II regions are found both throughout the late-type galaxies and in the very outskirts of early-type members. Considering the Dorado group as a whole, we notice that the $\mathrm{H} \alpha+[\mathrm{N}$ II $]$ equivalent width, a measure of the specific star formation, increases with morphological type from early- to latetype members, although it remains lower than that observed in similar surveys of spiral galaxies. The star formation rate of the spiral members is in the range of what is observed in similar galaxies surveys (James et al. 2004). However, in three spiral galaxies, NGC 1536, PGC 75125, and IC 2058, the star formation rate is well below the median for their morphological classes. Conversely, the star formation rate of some early-type members tends to be higher than the average derived from $\mathrm{H} \alpha+[\mathrm{N}$ II] surveys of this morphological family.

Conclusions. We detected $\mathrm{H} \alpha+[\mathrm{N}$ II] in all the early-type galaxies observed and half of them show $\mathrm{H}$ II regions in well-shaped rings as well as in their outskirts. These findings suggest that early-type galaxies in this group are not dead galaxies: their star formation has not yet shut down. Mechanisms such as gas stripping and gas accretion through galaxy-galaxy interaction seem relevant in modifying star formation in this evolutionary phase of Dorado.

Key words. galaxies: elliptical and lenticular, $\mathrm{cD}$ - galaxies: spiral - galaxies: ISM - galaxies: interactions - galaxies: evolution

\section{Introduction}

Driven by gravitation, groups and their member galaxies co-evolve. During group evolution, the properties of galaxies are significantly transformed, as we are starting to learn from colour-magnitude diagrams (CMDs) which provide a colour snapshot of this process (Balogh et al. 2004; Baldry et al. 2004; Schawinski et al. 2007; Kaviraj et al. 2007). In CMDs, blue versus red galaxy populations trace the transition from an active to a more evolved or even passive phase (Marino et al. 2010, 2013, 2016; Rampazzo et al. 2018). Member galaxies modify

* Data set are only available at the CDS via anonymous ftp to cdsarc.u-strasbg.fr $(130.79 .128 .5)$ or via http://cdsarc. u-strasbg.fr/viz-bin/cat/J/A+A/643/A176 both their star formation rate and morphology, from star forming late-type galaxies (LTGs) to early-type (ETGs; i.e. ellipticals and S0 galaxies (S0s)), quenching their star formation (Mazzei et al. 2014b). Several physical mechanisms are believed to play a role in this galaxy transformation, according to the richness of the surrounding environment (see e.g. Boselli \& Gavazzi 2006, 2014). As velocity dispersions of galaxies in groups are comparable to the velocity dispersion of stars in individual galaxies, both interactions and merging mechanisms are more favored in groups than in clusters (Mamon 1992). Mergers can transform LTGs into ETGs, that is both S0s and ellipticals (see e.g. Toomre \& Toomre 1972; Barnes 2002; Mazzei et al. 2014a, 2019) and quench star formation (SF) by ejecting the interstellar medium via starburst-, AGN-, or shock-driven winds (see e.g. Di Matteo 2015, and references therein). 
Tal et al. (2009) presented a deep imaging study - reaching $\mu_{V}=27.7 \mathrm{mag} \operatorname{arcsec}^{-2}-$ of a complete sample of luminous ETGs $\left(M_{B}<-20\right)$ at distances of $15-50 \mathrm{Mpc}$ selected from the Tully (1988) catalogue of nearby galaxies. Tal et al. find that $73 \%$ of them show tidal disturbance signatures in their stellar bodies. Concerning the relation between gravitational interaction signatures and the galaxy environment, these latter authors find that galaxies in clusters are less perturbed than group and field galaxies. Tal et al. (2009) concluded that ETGs in groups and low-density environments continue to grow at the present day through mostly dry mergers involving little star formation. More recently, Rampazzo et al. (2020) found that $\approx 60 \%$ of isolated ETGs display shell structures, an unambiguous merging signature, extending the view of intense galaxy transformation to very low-density environments.

In LTGs, the H $\alpha$ flux is directly connected to the star formation (SF) but this is not the case for ETGs. When $\mathrm{H} \alpha$ started to be widely detected in the central regions of ETGs using both spectroscopy (Heckman 1980; Phillips et al. 1986) and imaging (Goudfrooij et al. 1994b,a), several mechanisms were suggested to be at the origin of the emission in these galaxies, which are considered "red and dead". The $\mathrm{H} \alpha$ extended emission was thought to be connected with the hot gas phase giving rise to the strong X-ray emission in ETGs (see e.g. Trinchieri et al 1997, and references therein). The nuclei of most ETGs show low- ionisation nuclear emission region (LINER) properties, which could explain the $\mathrm{H} \alpha$ emission in the central regions. The role of post asymptotic giant branch (PAGB) stars has also been emphasised in cases where the emission is more extended (see e.g. Annibali et al. 2010). Both GALEX (Jeong et al. 2009; Marino et al. 2011a) and Spitzer (see e.g. Panuzzo et al. 2007, 2011; Vega et al. 2010; Rampazzo et al. 2013, and references therein) observations have increasingly demonstrated that SF can also be found in ETGs and can be at the origin of the $\mathrm{H} \alpha$ emission both in the centre and in the outer regions, often characterised by resonance rings. ETGs have been shown to often be the fuel of the SF (see e.g. Serra et al. 2012, and references), a phenomenon also often revealed in H I. Recently, a $\mathrm{H} \alpha$ survey of 147 ETGs, out of 260 in the ATLAS ${ }^{3 \mathrm{D}}$ sample, was performed by Gavazzi et al. (2018), with 92 ETGs being undetected. These 147 are gas-free systems that lack a disc and exhibit passive spectra, even in their nuclei. Most $(76 \%)$ of the remaining 55 ETGs from this sample are strong $\mathrm{H} \alpha$ emitters and were found to be associated with low-mass $\left(M * \approx 10^{10} M_{\odot}\right)$ S0 galaxies, showing conspicuous gas $\left(\mathrm{HI}+\mathrm{H}_{2}\right)$ content, extended stellar discs, and SF also in their nuclei.

In the above context, the study of the Dorado group offers the possibility to investigate accretion and merging events from the perspective of gas content. Dorado is a H I rich group, with a total H I mass of $3.5 \times 10^{10} M_{\odot}$, nearly half of which is concentrated in the spiral member NGC 1566 (Kilborn et al. 2005, 2009). These latter studies detected 13 galaxies including ETGs (NGC 1533, NGC 1543, NGC 1596). In addition, the H I distribution offers an indication of the strong interaction between Dorado members, which may re-fuel gas-poor systems such as ETGs. Ryan-Weber et al. (2003) revealed a vast system of H I around NGC 1533 with a tail connecting the galaxy to the IC2038/IC 2039 pair located several kiloparsecs away. Indeed, irrespective of their morphological classification, Dorado members show a significant fraction of galaxies, from the centre to the group periphery, showing either galaxy-galaxy interaction or merging signatures.

The UV-optical Dorado CMD (see Fig. 1 in Cattapan et al. 2019) is composed of both a red sequence, including several
ETGs, and a green valley populated by numerous intermediateluminosity galaxy members. Only NGC 1566, a bright "granddesign" spiral, is still located in the Blue Cloud. Therefore, with respect to the rich NGC 5486 group (Marino et al. 2016), Dorado is in an earlier and more active evolutionary phase (see Sect. 2) and offers a possibility to investigate the evolutionary mechanisms in action. Our study with GALEX of the early-type members, NGC 1533 and NGC 1543 (Marino et al. 2011b; Rampazzo et al. 2017), provide direct evidence of the presence of SF. Mazzei et al. (2014a,b) used smoothed particle hydrodynamic (SPH) simulations with chemo-photometric implementation to successfully reproduce the morphological, kinematical, and spectral energy distributions of two members of the Dorado group, NGC 1533 and NGC 1543, as a merger byproduct (Mazzei et al. 2019).

The present work is motivated by indications of SF and dissipation events reported for Dorado members in the current literature. In this paper we aim to investigate the SF of the Dorado members, both early- and late-type galaxies, via $\mathrm{H} \alpha+[\mathrm{N}$ II] narrow-band imaging observations. Several studies used $\mathrm{H} \alpha$ fluxes as an indicator of SF and calibrated the relation (see the review Kennicutt 1998). As a reference, we mention the $\mathrm{H} \alpha$ surveys of LTGs by James et al. (2004) and Kennicutt et al. (2009). James et al. (2004) found a strong correlation between total star formation rate (SFR) and Hubble type. The strongest SFR is found in isolated galaxies and occurs in Sc and Sbc types. The Dorado group was not included in the Kennicutt et al. (2008) study of the local volume. However, some $\mathrm{H} \alpha$ studies have been dedicated to NGC 1566, the brightest spiral member. Its $\mathrm{H} \alpha$ total luminosity, $L_{\mathrm{H} \alpha}$, was obtained by Hoopes et al. (2001) and Kennicutt et al. (2009). Roy \& Walsh (1986) studied the $\mathrm{H} \alpha$ emission in the outer NW arm of the galaxy. Uncalibrated $\mathrm{H} \alpha$ observations of IC 2058, an edge-on spiral spiral member, were performed by Rossa \& Dettmar (2003) with the aim of studying the $\mathrm{H} \alpha$ extra-planar distribution. Trinchieri et al. (1997) obtained a deep $\mathrm{H} \alpha+[\mathrm{N} \mathrm{II]} \mathrm{image} \mathrm{of} \mathrm{NGC} \mathrm{1553,} \mathrm{the} \mathrm{brightest} \mathrm{among}$ ETGs in the group.

Our work is based on $\mathrm{H} \alpha+[\mathrm{N}$ II] images obtained at the Irénée du Pont $2.5 \mathrm{~m}$ and Henrietta Swope $1 \mathrm{~m}$ telescopes at Las Campanas Observatory (Chile). The paper is organised as follows. In Sect. 2 we describe the Dorado group and suggest it is still undergoing a strong transition phase. Appendix A provides the member list adapted from the dynamical studies of Kourkchi \& Tully (2017) and Firth et al. (2006). In Sect. 3 we present $\mathrm{H} \alpha+[\mathrm{N} \mathrm{II}]$ observations and the reduction performed. The analysis of the $\mathrm{H} \alpha+[\mathrm{N}$ II] data is presented in Sect. 4. In Sect. 5 we discuss the morphology of the $\mathrm{H} \alpha+[\mathrm{N} \mathrm{II}]$ emission in each individual galaxy. A comparison of our results with the literature is provided in Appendix B. The SFR is discussed as a function of member morphological type. In the context of group evolution, Sect. 6 discusses the SFR in the group substructures.

\section{Dorado group: an overview}

Before presenting our observations we describe the structure of the group, also discussing the membership of the galaxies that form its backbone, namely our targets.

The Dorado group $(\mathrm{RA}=64.3718$ [deg], Dec $=-55.7811$ [deg]) extends for several degrees in the southern hemisphere (see e.g. Firth et al. 2006; Kourkchi \& Tully 2017, and reference therein; Fig. 1). In this paper we assume that members are at the group distance of 17.69 Mpc (Kourkchi \& Tully 2017). The group includes both bright ETGs and LTGs. Its structure is quite loose and clumpy, meaning that the two giant 


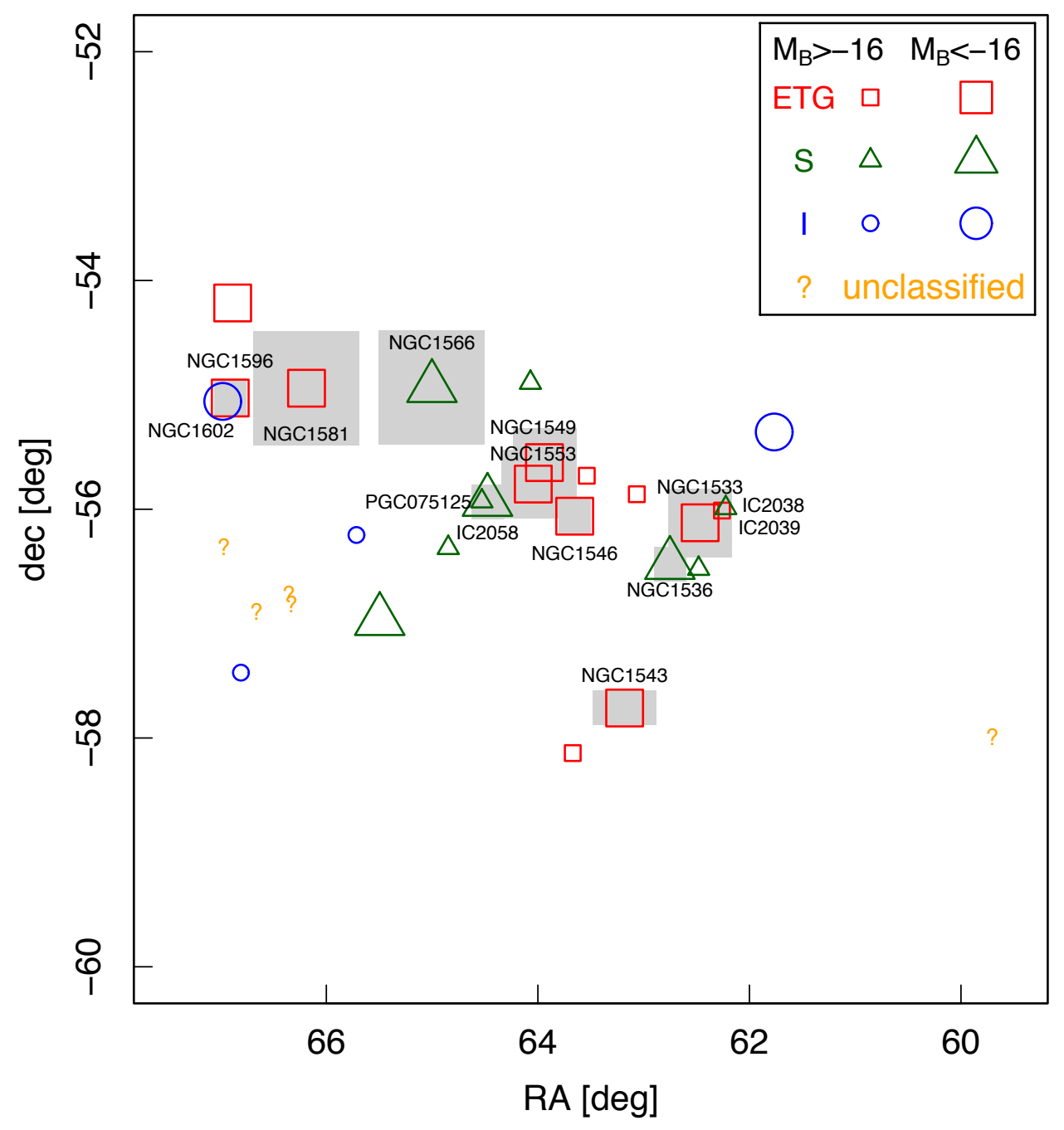

Fig. 1. Projected distribution of the Dorado group member galaxies (see Appendix A). Galaxies labeled are those investigated in the present $\mathrm{H} \alpha+[\mathrm{N}$ II] study. Galaxies are indicated according to their $B$-band absolute magnitude and morphological type. Fields covered by the du Pont and Swope pointings, listed in Table 1, are indicated in grey. galaxies NGC 1566, a grand-design spiral, and NGC 1553, an SO galaxy, have been considered as the centres of two homonymous but independent groups. In this sense, different member lists have been compiled with different selection criteria. The NGC 1566 group is composed of 18 member galaxies according to Huchra \& Geller (1982), while Garcia (1993) and Brough et al. (2006) consider the group to be made up of 6 and 4 galaxy members, respectively. Makarov \& Karachentsev (2011) reviewed the NGC 1553 group membership, including 29 members. The Dorado faint galaxy population is still poorly defined. A large population of dwarf galaxies is expected in groups dominated by ETGs, as is the case of Dorado. From a Canada-FranceHawaii Telescope (CFHT) Megacam campaign, Tully (2015) reported that these groups have larger dwarf-to-giant ratios with respect to groups dominated by LTGs (considering giants as those with $M_{r}<-17$ and dwarfs with $-17<M_{r}<-11$, $\left.\mathrm{H}_{0}=75 \mathrm{~km} \mathrm{~s}^{-1} \mathrm{Mpc}^{-1}\right)$. An attempt to map the Dorado faint galaxy population was made by Carrasco et al. (2001) but without determining their redshift.

Firth et al. (2006) redefined the Dorado members on the basis of the group structure and its dynamical properties. The Firth et al. (2006) Dorado backbone is composed of 20 members starting from the 79 galaxies, including a few dwarfs that Ferguson \& Sandage (1990) identified and catalogued in the Dorado region. The Dressler \& Shectman (1988) test applied to the group candidates by Firth et al. (2006) provided a 2D view of the group clumpiness. Furthermore, Firth et al. (2006) performed a crossing-time test checking for virialisation and found an indication that the group is still undergoing dynamical relaxation.

Recently Kourkchi \& Tully (2017) revised the group structure, findings that 31 galaxies belong to Dorado according to their systemic radial velocities and membership criteria from Tully (2015). The group velocity dispersion is $242 \mathrm{~km} \mathrm{~s}^{-1}$ (Kourkchi \& Tully 2017). The average recession velocity is $\left\langle V_{\text {hel }}\right\rangle=1230 \pm 89 \mathrm{~km} \mathrm{~s}^{-1}$. The $2^{\circ}$ turnaround radius and the virial radius are $0.653 \mathrm{Mpc}$ and $0.654 \mathrm{Mpc}$, respectively. The group virial mass weighted on virial radius is $3.50 \times 10^{13} M_{\odot}$ (formulæ and definitions are provided by Kourkchi \& Tully 2017 and Tully 2015).

The list of candidate members adopted in this paper is presented and discussed in Appendix A. We considered 31 member candidates: 30 from Kourkchi \& Tully (2017) list excluding 2MASXJ04105983-5628496, plus PGC 75125 from Firth et al. (2006). Figure 1 shows the distribution in RA(J2000) and Dec (J2000) of the Kourkchi \& Tully (2017) members. Appendix A provides further information about the group. Figure A.1 shows the morphological type distribution, which includes both ETGs and LTGs, and the velocity distribution. The galaxy population is dominated by the two giant ETGs, NGC 1549 and NGC 1553, the pair AM 0414-554/AM 0415-555 (Arp \& Madore 1987) located roughly in the projected group centre, and the bright 
spiral, NGC 1566, at the group periphery. Iovino (2002) suggested that the pair NGC 1549/NGC 1553 is part of SCG 04145559, a compact group that also includes a spiral, IC 2058, and an S0, NGC 1546.

NGC 1549 and NGC 1553, in the group projected centre, show a wide system of shells and ripples (Malin \& Carter 1983) whose origin is connected to merging and/or accretion episodes (Dupraz \& Combes 1987; Weil \& Hernquist 1993; Mancillas et al. 2019). While NGC 1553 has a normal rotation curve, NGC 1549 also shows a velocity gradient along its minor axis (Rampazzo 1988). NGC 1533 and NGC 1543, both S0s, show strong ring-like FUV emission (Marino et al. 2011b,a). Rampazzo et al. (2017) further investigated NGC 1533 and NGC 1543 using Swift-UVOT (Roming et al. 2005) in $W 1$, $M 2$, and $W 2$ filters, confirming the presence of a young stellar population. Mazzei et al. (2014a,b, 2019) by matching the global properties of NGC 1533 and NGC 1543, derive their age and that of mergers (major and minor respectively) which drive their evolution. Cattapan et al. (2019) deep surface photometry unveiled strong interaction signatures in NGC 1533, IC 2038, and IC 2039. NGC 1536 (AM 0409-563) is a late-type galaxy showing a clear asymmetry in its structure, likely the result of an ongoing interaction. NGC 1596 is a lenticular galaxy interacting with NGC 1602, an LTG. In NGC 1596, the ionised gas counter-rotates with respect to stars (Bureau \& Chung 2006). Chung et al. (2006) suggested that the origin of the counterrotating gas is the interaction between the two galaxies that are embedded in a common H I cloud.

The presence of AGN-like activity has only been investigated in some of the Dorado members. Annibali et al. (2010) found that NGC 1533 and NGC 1553 are LINERs in their nuclear region (these authors investigated the region $r_{e} / 8-$ $r_{e} / 2$, but the classification in their Table 4 is relative to $\left.r_{e} / 16\right)$. Rampazzo et al. (2013) studied the nuclear regions of NGC 1533, NGC 1549 and NGC 1553 in the mid-infrared with Spitzer, unveiling polyaromatic hydrocarbons (PAHs) with anomalous ratios in their spectra. These features have been interpreted as a relic of past star formation activity (see e.g. Vega et al. 2010).

The high fraction of signatures of galaxy-galaxy encounters, accretions, and merging suggest that Dorado members are going through a deep transforming phase, revealed by the (NUV $-r$ ) versus $M_{r}$ CDM (Cattapan et al. 2019). Many ETGs have already reached the red sequence, while other galaxies are still transforming in the green valley. For the former, our objective is to test whether or not their star formation is completely quenched. For galaxies in the green valley, we aim to check how their SFR compares with that of galaxies of similar morphological type.

\section{Observations and reduction}

In this section we describe our $\mathrm{H} \alpha+[\mathrm{N} \mathrm{II}]$ data set and the reduction techniques we applied. The filter pass band includes [N II] emission lines (Fig. 2). The SFR of galaxies is derived applying a correction to the $\mathrm{H} \alpha$ emission, following the recipe of Lee et al. (2009); see Sect. 5.3.

A large fraction of Dorado members are dwarfs (see Appendix A.1). The separation of most of them from other members would have required a specific pointing. We decided to concentrate our efforts on bright galaxies for two reasons: (1) they have nearby companions and (2) they are well-studied galaxies, that is, additional information about their evolution (structure, kinematics, spectroscopic studies) is already available in

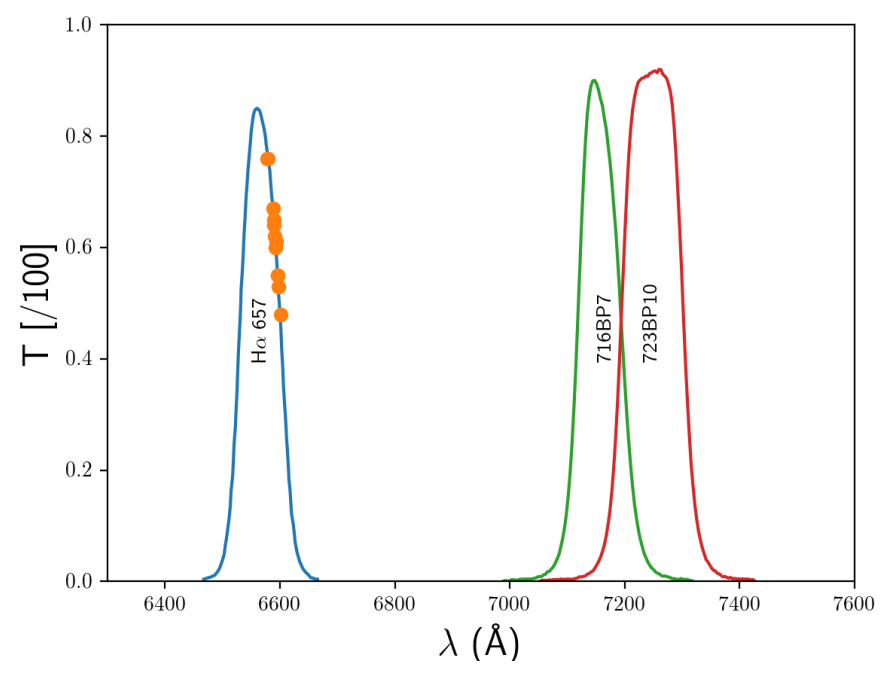

Fig. 2. Transmission profiles of the narrow band filters on and off the $\mathrm{H} \alpha$ line. Observed galaxies are indicated as dots on the $\mathrm{H} \alpha+[\mathrm{N}$ II] filter at the wavelength given by their heliocentric recession velocity.

the literature. Considering that we have lost one and a half nights because of bad weather conditions, the final sample resulted of 14 galaxies (13 in Kourkchi \& Tully 2017 plus PGC 75125 in the Firth et al. 2006 list).

\section{1. $\mathrm{H} \alpha+[\mathrm{N} I I]$ observations}

$\mathrm{H} \alpha+[\mathrm{N} \mathrm{II}]$ images were obtained using the direct CCD Camera at the du Pont $2.5 \mathrm{~m}$ and at Swope $1 \mathrm{~m}$ telescopes at Las Campanas Observatory (LCO, Chile). Observations cover the period between November 30 and December 7, 2018. Table 1 reports the details of the observing runs.

The SITe2K CCD at the du Pont telescope covers a field of view (FOV) of $8.8 \mathrm{arcmin}^{2}$ with a spatial scale of $0.259 \mathrm{pix}^{-1}$, while the E2V CCD231-84 CCD at the Swope telescope has a larger FOV of $29.7 \times 29.8 \mathrm{arcmin}^{2}$ with a spatial sampling of 0 ". $435 \mathrm{px}^{-1}$. Table 1 shows that some du Pont observations are composed of a mosaic of frames for the bright Dorado members, and a single frame for less extended galaxies. NGC 1566 and NGC 1581 were observed with a single, wide field image at the Swope telescope.

$\mathrm{H} \alpha+[\mathrm{N} \mathrm{II}]$ and nearby continuum images were obtained with the following filters: on-band \#657 (FWHM = 74 $\AA$ ), and offbands \#716 (FWHM = 77 $\AA$ ) and \#723 (FWHM=109 $\AA)$. The $\mathrm{H} \alpha+[\mathrm{N} \mathrm{II}] \# 657$ contains the [N II] $\lambda \lambda 6548,6584$ emission lines, while the \#716 filter can be contaminated by [Ar III] $\lambda 7136$, which is nevertheless a weak line, and \#723 is almost completely free of emission lines.

The strategy of the observations consisted of taking $3 \times 900 \mathrm{~s}$ (at the du Pont telescope) and $3 \times 1200$ s (at the Swope telescope) images for each filter on-band and off-band. The off-band image is obtained after each on-band image in order to minimise the seeing variations and to facilitate the continuum subtraction. For some of the galaxies, more than one pointing was required to cover the desired FOV. The seeing changed from night to night between 0 '.9 and 1 '!6, but it was rather stable within each single night.

\section{2. $H \alpha+[N I I]$ reduction}

Data reduction was performed using the IRAF reduction package (Tody 1986). Raw scientific data were corrected for 
Table 1. H $\alpha$ narrow band observations.

\begin{tabular}{|c|c|c|c|c|c|c|c|}
\hline $\begin{array}{l}\text { Field } \\
\text { ID }\end{array}$ & Telescope & Observing date & $\begin{array}{l}\text { Exp. time } \\
{[\mathrm{s}]}\end{array}$ & $\begin{array}{l}\text { Filter } \\
\text { Ident. }\end{array}$ & $\begin{array}{c}\text { Scale } \\
{\left[\operatorname{arcsec} \mathrm{px}^{-1}\right]}\end{array}$ & $\begin{array}{c}\text { Seeing } \\
{[\operatorname{arcsec}]}\end{array}$ & Notes \\
\hline \multirow[t]{2}{*}{ NGC 1533} & du Pont & Nov. 30/Dec. 1, 2018 & $900 \times 3$ & 716 & 0.259 & $0.94 \pm 0.19$ & 5 frames, $\mathrm{FoV}=17: 7^{2}$ \\
\hline & & & $900 \times 3$ & 657 & 0.259 & & 5 frames, $\mathrm{FoV}=17 \cdot 7^{2}$ \\
\hline \multirow[t]{2}{*}{ NGC 1536} & du Pont & Dec. 5, 2018 & $900 \times 3$ & 723 & 0.259 & $1.03 \pm 0.05$ & 1 frame $\mathrm{FoV}=8.85^{2}$ \\
\hline & & & $900 \times 3$ & 657 & 0.259 & & 1 frame $\mathrm{FoV}=8: 85^{2}$ \\
\hline \multirow[t]{2}{*}{ NGC 1543} & du Pont & Dec. $3 / 5,2018$ & $900 \times 3$ & 723 & 0.259 & $0.96 \pm 0.09$ & 2 frames, $\mathrm{FoV}=17.7 \times 8.85$ \\
\hline & & & $900 \times 3$ & 657 & 0.259 & & 2 frames, $\mathrm{FoV}=17.7 \times 8.85$ \\
\hline \multirow[t]{2}{*}{ NGC 1546} & du Pont & Dec. 5, 2018 & $900 \times 3$ & 723 & 0.259 & $1.26 \pm 0.05$ & 1 frame $\mathrm{FoV}=8{ }^{\prime} 85^{2}$ \\
\hline & & & $900 \times 3$ & 657 & 0.259 & & 1 frame $\mathrm{FoV}=8^{\prime} .85^{2}$ \\
\hline \multirow[t]{2}{*}{ NGC 1549} & du Pont & Dec. 2, 2018 & $900 \times 3$ & 716 & 0.259 & $0.95 \pm 0.16$ & 4 frames, $\mathrm{FoV}=17: 7^{2}$ \\
\hline & & & $900 \times 3$ & 657 & 0.259 & & 4 frames, $\mathrm{FoV}=17: 7^{2}$ \\
\hline \multirow[t]{2}{*}{ NGC 1553} & du Pont & Dec. 2, 2018 & $900 \times 3$ & 723 & 0.259 & $0.90 \pm 0.06$ & 4 frames, $\mathrm{FoV}=17: 7^{2}$ \\
\hline & & & $900 \times 3$ & 657 & 0.259 & & 4 frames, $\mathrm{FoV}=17: 7^{2}$ \\
\hline \multirow{2}{*}{ IC 2058} & du Pont & Dec. 3, 2018 & $900 \times 3$ & 723 & 0.259 & $1.54 \pm 0.05$ & 1 frame $\mathrm{FoV}=8{ }^{\prime} 85^{2}$ \\
\hline & & & $900 \times 3$ & 657 & 0.259 & & 1 frame $\mathrm{FoV}=8^{\prime} .85^{2}$ \\
\hline \multirow[t]{2}{*}{ NGC 1566} & Swope & Dec. 6, 2018 & $1200 \times 3$ & 723 & 0.435 & $1.60 \pm 0.03$ & 1 frame FoV $=29 ! 7 \times 29.8$ \\
\hline & & & $1200 \times 3$ & 657 & 0.435 & & 1 frame FoV $=29 ! 7 \times 29 ! 8$ \\
\hline \multirow[t]{2}{*}{ NGC 1581} & Swope & Dec. 7, 2018 & $1200 \times 3$ & 723 & 0.435 & $1.67 \pm 0.04$ & 1 frame $\mathrm{FoV}=29.7 \times 29{ }^{\prime} .8$ \\
\hline & & & $1200 \times 3$ & 657 & 0.435 & & 1 frame FoV $=29 ! 7 \times 29.8$ \\
\hline \multirow[t]{2}{*}{ NGC 1596} & du Pont & Dec. 5, 2018 & $900 \times 3$ & 723 & 0.259 & $1.63 \pm 0.01$ & 1 frame $\mathrm{FoV}=8.85^{2}$ \\
\hline & & & $900 \times 3$ & 657 & 0.259 & & 1 frame $\mathrm{FoV}=8.85^{2}$ \\
\hline
\end{tabular}

Notes. Field ID refers to the main galaxy in the field. Column 2 reports the LCO Telescope used. Columns 3 and 4 report the observing date and the total reduced exposure time. Columns 5 and 6 quote the off-on $\mathrm{H} \alpha$ filters and the image scale. The FWHM of the filters H $\alpha 657,716 \mathrm{BP} 7$ and $723 \mathrm{BP} 10$ are $77 \AA, 77 \AA$ and $109 \AA$, respectively (see also Fig. 2). In Col. 7 the average seeing measured in the frames reported in the notes of column 8 which provides also the total field of view covered. Images are obtained using as GAIN $=1.54 \mathrm{e}^{-} \mathrm{ADU}^{-1}$ and READOUT $=6.5 \mathrm{e}^{-}$for the SITe2K CCD @ du Pont; GAIN = 1.040 $\mathrm{e}^{-} \mathrm{ADU}^{-1}$ and READOUT =3.0 $\mathrm{e}^{-}$for the E2V CCD231-84 CCD @ Swope.

overscan. Flat-field correction was applied using twilight sky flats acquired during the observing run. Each image was background subtracted, normalised to $1 \mathrm{~s}$ exposure time, and corrected for airmass by means of the CTIO atmospheric extinction curve.

Each off-band image was first corrected for the different transmission curves of \#716 and \#723 with respect to the onband $\mathrm{H} \alpha \# 657$ filter, and then registered to the on-band image with the IRAF task IMALIGN. In addition, the PSFMATCH task was applied to take into account the small seeing variations before proceeding with the continuum subtraction. The continuumsubtracted, calibrated individual $\mathrm{H} \alpha+[\mathrm{N}$ II] images were combined to finally produce a single averaged image per pointing. Multiple pointings, when available, were combined to compose the mosaic using SWarp (Bertin et al. 2002).

With the exception of some bright stars, the subtraction procedure was generally successful, as most of the stars disappeared with small or even no residuals. Saturated stars cannot obviously be well subtracted and leave large residuals on the final images.

\subsection{Flux calibration}

Photometric standard stars LTT 1788, LTT 3218, LTT 3864, LTT 4816, and Feige 110 observed during the nights were used for flux calibration. Raw images were processed as explained above up to airmass correction. In order to calculate the flux calibration constants, the PHOT task was used to measure the instrumental fluxes. These fluxes were then compared to the real fluxes obtained by multiplying the spectrum of each standard star (extracted from IRAF database) for the transmission curve of the on-band \#657 filter.

\section{Data analysis}

Before carrying out measurements, the background of each continuum subtracted image was inspected and carefully re-fitted and re-subtracted with the IRAF task IMSURFIT if necessary. This is a delicate point, because even very small gradients can produce spurious fluxes when large areas of the image are integrated. To estimate the depth of the newly processed images, we measured the average surface brightness standard deviation, which ranges between 3 and $5 \times 10^{-17} \mathrm{erg} \mathrm{cm}^{-2} \mathrm{~s}^{-1} \operatorname{arcsec}^{-2}$.

We applied the IRAF task ELLIPSE to the off-band images, keeping centre, ellipticity, and position angle fixed in order to obtain concentric apertures. The ellipse parameters, reported in Table 2 (columns 3-5) were chosen on the basis of the outer isophotes of the images in the off-band continuum. Residuals of stars, particularly bright and saturated stars, were carefully masked. Figures 3 and 4 show on-band and off-band images, respectively, of the triplet (Cattapan et al. 2019) composed of the spiral IC 2038 and the two early-type galaxies IC 2039 and NGC 1533.

Once the off-band images were fitted, the same ellipse was applied to the on-band continuum-subtracted ones. Following the examples and suggestions by James et al. (2004) we calculated the $\mathrm{H} \alpha+[\mathrm{N} \mathrm{II}]$ equivalent width $(\mathrm{EW})$ profiles, and the total flux within the largest apertures showing emission up to $1 \sigma$ level. Finally, the fluxes were corrected for the transmission of the on-band $\mathrm{H} \alpha$ filter at the wavelength corresponding to the radial velocity of each single galaxy, as given in Table A.1. The $3 \sigma$ error associated to each measure is calculated using the sky background measured in five independent regions outside the ellipse whose parameters are reported in Table 2. 
Table 2. Parameters used to measure the $\mathrm{H} \alpha+[\mathrm{NII}]$ flux and its EW.

\begin{tabular}{lccccc}
\hline \hline $\begin{array}{l}\text { ID } \\
\text { source }\end{array}$ & $\epsilon$ & $\begin{array}{c}\text { PA } \\
{\left[{ }^{\circ}\right]}\end{array}$ & $\begin{array}{c}a \\
{[\operatorname{arcsec}]}\end{array}$ & $\begin{array}{c}F(\mathrm{H} \alpha+\mathrm{NII}) \\
{\left[\times 10^{-14} \mathrm{erg} \mathrm{cm}^{-2} \mathrm{~s}^{-1}\right]}\end{array}$ & $\begin{array}{c}E W \\
{[\mathrm{~nm}]}\end{array}$ \\
\hline IC 2038 & 0.63 & 150 & 51.3 & $11.0 \pm 2.9$ & $2.2 \pm 0.7$ \\
IC 2039 & 0.05 & 0 & 19.4 & $2.8 \pm 1.1$ & $0.7 \pm 0.3$ \\
NGC 1533 & 0.05 & 0 & 89.2 & $182.5 \pm 7.0$ & $0.9 \pm 0.1$ \\
NGC 1536 & 0.27 & 165 & 43.0 & $45.4 \pm 1.6$ & $1.5 \pm 0.2$ \\
NGC 1543 & 0.05 & 0 & 88.0 & $172.9 \pm 17.7$ & $0.8 \pm 0.1$ \\
NGC 1546 & 0.19 & 145 & 54.3 & $93.1 \pm 4.0$ & $1.0 \pm 0.1$ \\
NGC 1549 & 0.05 & 0 & 73.0 & $192.7 \pm 10.5$ & $0.5 \pm 0.1$ \\
NGC 1553 & 0.25 & 145 & 135.6 & $545.1 \pm 65.2$ & $0.7 \pm 0.1$ \\
IC 2058 & 0.75 & 18 & 79.1 & $33.3 \pm 3.6$ & $2.0 \pm 0.2$ \\
PGC 75125 & 0.36 & 20 & 15.8 & $3.3 \pm 0.3$ & $2.2 \pm 0.2$ \\
NGC 1566 & 0.10 & 20 & 227.0 & $1246.7 \pm 125.1$ & $1.8 \pm 0.2$ \\
NGC 1581 & 0.50 & 82 & 35.7 & $50.3 \pm 4.3$ & $1.1 \pm 0.2$ \\
NGC 1596 & 0.61 & 20 & 69.4 & $119.7 \pm 6.5$ & $0.8 \pm 0.1$ \\
NGC 1602 & 0.26 & 85 & 56.5 & $97.6 \pm 6.9$ & $4.4 \pm 0.5$ \\
\hline
\end{tabular}

Notes. Column 1 provides the source identity. Columns 2-4: are the ellipticity, $\epsilon$, the Position Angle (PA measured north to east) and the semi-major axis, $a$ of the ellipse within which the fluxes were measured respectively. Column 5 is $\mathrm{H} \alpha+[\mathrm{N} \mathrm{II}]$ flux measured within the elliptical aperture and the $3 \sigma$ error. Column 6 is the EW within the ellipse.

We remind the reader that fluxes are not corrected for internal reddening because the value of the extinction is unknown. Nonetheless it is worth noting that reddening and [N II] contamination change the $\mathrm{H} \alpha$ flux in opposite directions, such that they can even compensate each other but both effects cannot be quantified with the present data. This uncertainty does not apply to the EW, which is almost independent of reddening. The final $\mathrm{H} \alpha+[\mathrm{N} \mathrm{II}]$ fluxes and EW are reported in Table 2.

\section{Results}

We now present the results of our analysis for each single galaxy. In Appendix B we instead compare our results with the available literature.

\subsection{Continuum versus $H \alpha+[N$ II] morphology: individual notes}

We report below some notes about the $\mathrm{H} \alpha+[\mathrm{N} \mathrm{II}]$ emission in our targets with particular attention to asymmetries or correlations with features present in the continuum image and to resonance structures. We present and discuss two-colour images (continuum in green and $\mathrm{Ha}+[\mathrm{NII}]$ in red) which emphasise the morphology of $\mathrm{H} \alpha$ emission regions.

IC 2038/IC 2039. These two galaxies are classified Scd $($ Type $=7.0 \pm 0.5)$ and E-S0 $($ Type $=-3.1 \pm 0.7)$, respectively. According to Cattapan et al. (2019) the galaxies show clear signatures of interaction. The $\mathrm{H} \alpha$ distribution of IC 2038, shown in Fig. 3, is clumpy and marks the presence of $\mathrm{H}$ II regions, without any obvious arm structure. H II regions are distributed along the galaxy body which appears slightly elongated towards the companion galaxy in the SE direction (see Cattapan et al. 2019, their Fig. 2). In IC 2039, the $\mathrm{H} \alpha+[\mathrm{N} \mathrm{II}]$ emission is diffuse and smooth, without $\mathrm{H}$ II regions.

NGC 1533. This galaxy is classified E-S0 with a bar (Type = $-2.5 \pm 0.6$ ) by HyperLeda and (RL)SB0 ${ }^{0}$ by Comerón et al. (2014), that is, the galaxy has an outer ring, a lens, and an inner bar. H II regions in Fig. 4, labelled A and B, correspond to the rings clearly revealed in far-ultraviolet (FUV) by GALEX (see e.g. Werk et al. 2010; Marino et al. 2011c; Rampazzo et al. 2017).

NGC 1536. This galaxy is classifed as SBc (Type $=4.6 \pm$ 1.8) in HyperLeda. In the continuum image the galaxy shows a thin bar in the central region and irregular arms with signatures of interaction. The $\mathrm{H} \alpha+[\mathrm{N} \mathrm{II}]$ emission follows roughly the structures seen in the continuum (Fig. C.1). H II regions are asymmetrically distributed with respect to the inner bar. These regions develop along the NW arm and are scattered within the south galaxy body.

NGC 1543. This galaxy is considered a S0 with bar and a ring (Type $=-2.0 \pm 0.8$ ) in HyperLeda. The $\mathrm{H} \alpha+[\mathrm{N} \mathrm{II}]$ emission follows the bar in the galaxy central region (see Fig. C.2). The $\mathrm{H} \alpha$ emission is concentrated in some H II complexes, labelled A, $\mathrm{B}$, and $\mathrm{C}$ in Fig. C.2, along the southeast edge of the outer ring. These SF areas are clearly visible in FUV GALEX observations (Marino et al. 2011b,a; Rampazzo et al. 2017).

NGC 1546. This galaxy, considered a S0-a (Type $=-0.4 \pm$ 1.7) in Comerón et al. (2014), is classified E(b)3/(R')SA(r)ab, that is, it could be a $3 \mathrm{D}$ ETG or a SA with an inner ring. The continuum image in Fig. C.3 shows a small bulge embedded in a strongly inclined disc (53.3 degree in Hyperleda). The $\mathrm{H} \alpha+[\mathrm{N}$ II] emission shows a flocculent structure inside the disc. The H II regions, which are emphasised in the two-colour image, follow the inner and outer rings evidenced by Comerón et al. (2014). A strong dust lane is visible in the SW ridge of the galaxy.

NGC 1549. This galaxy is considered in HyperLeda to be a true elliptical (Type $=-4.3 \pm 0.9$ ). The $\mathrm{H} \alpha+[\mathrm{N} \mathrm{II}]$ emission in Fig. C.4 (top right panel) shows an amorphous distribution like that of the continuum (top left panel). No H II regions are visible.

NGC 1553. This galaxy is classified S0 with a ring (Type $=-2.3 \pm 0.6$ ). Comerón et al. (2014) classified it $\mathrm{SA}\left(\mathrm{rl}, \mathrm{nr}^{\prime} \mathrm{l}\right) 0^{+}$, that is, two rings are present in this lenticular galaxy. For NGC 1553, 75\% of the $\mathrm{H} \alpha+[\mathrm{N}$ II $]$ flux is measured within an ellipse of $60^{\prime \prime}$ of semi-major axis, containing the core and the ring visible in Fig. C.5, which is a relatively small central region with respect to the size of the ellipse reported in Table 2. No H II regions are revealed in the galaxy (see, however, Sect. 6.1). Trinchieri et al. (1997) show a $\mathrm{H} \alpha+[\mathrm{N} \mathrm{II}]$ image of the galaxy (their Fig. 11, right panel) but no flux is provided in that paper. At faint levels (the authors smoothed the image to enhance features), the morphology in their image shows a noisy spiral arm, in the southeast direction, which is not detected in our observations.

IC 2058. This galaxy is an edge-on spiral ( $\mathrm{Scd}$, Type $=$ $6.6 \pm 0.9) . \mathrm{H} \alpha+[\mathrm{N} \mathrm{II}]$ emission is revealed along the plane of the galaxy and no obvious bulge is visible (see Fig. C.6). Uncalibrated $\mathrm{H} \alpha$ observations of this galaxy performed by Rossa \& Dettmar (2003) did not reveal any extra-planar $\mathrm{H} \alpha$ emission in the halo of this spiral galaxy. Figure C.6 shows that $\mathrm{H}$ II regions are indeed distributed all along its disc.

PGC 75125. This dwarf galaxy is a companion of IC 2058 according to Firth et al. (2006). The two galaxies are nearby in projection and have a heliocentric velocity difference $\Delta V_{\text {hel }}=$ $26 \mathrm{~km} \mathrm{~s}^{-1}$. According to HyperLeda, the galaxy morphological type is $T=5.0 \pm 3.0$. In the continuum image, the galaxy structure appears boxy and without spiral arms. An elongated, distorted $\mathrm{H} \alpha+[\mathrm{N} \mathrm{II}]$ distribution (Fig. C.7) appears in the outer 


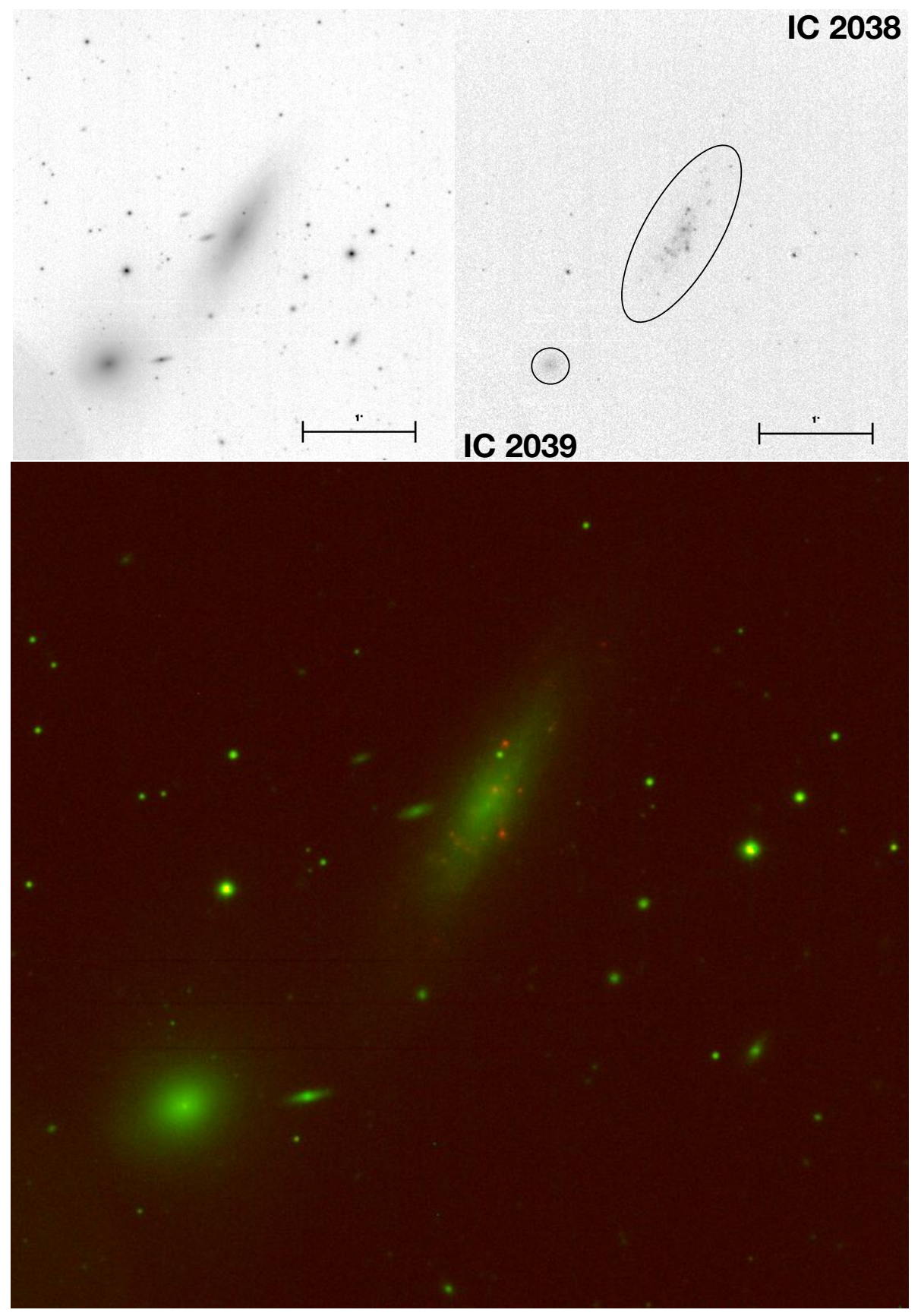

Fig. 3. Pair IC2038 (NW) and IC2039 (SE) in continuum (top left panel) and in the $\mathrm{H} \alpha+[\mathrm{NII}]$ light (top right panel). The image size is $4^{\prime} \times 4^{\prime}$. North is towards the top, and east is to the left. Residuals of stars have been masked. The ellipse, whose parameters are given in Table 2, encloses the integration area. The bottom panel shows a two-colour image of the pair (continuum in green and $\mathrm{H} \alpha+[\mathrm{N} \mathrm{II}]$ in red) that highlights a large numbers of brights HII regions. regions of this galaxy. The $\mathrm{H} \alpha+[\mathrm{N}$ II] emission could mark the (warped) disc of the galaxy. We suggest this galaxy is likely a late S0.

NGC 1566. This is a grand-design barred galaxy classified as a $\mathrm{SABb}$ (Type $=4.0 \pm 0.2$ ) in the HyperLeda catalogue. According to Comerón et al. (2014), who classified the galaxy (R'1)SB(r'l, s,nb)b, there are two rings of which we do not find any evidence in the $\mathrm{H} \alpha+[\mathrm{N} \mathrm{II}]$ image (Fig. C.8). NGC 1566 is asymmetric (the southern part is more stretched than the northern one).

NGC 1581. This is an E-S0 galaxy (Type $=-2.9 \pm 0.8$ according HyperLeda). Figure C.9 shows an inner ring both in the continuum and in the $\mathrm{H} \alpha+[\mathrm{N} \mathrm{II}]$ images. Small bright H II regions are visible near the galaxy centre and in the inner ring, which is brighter in the west region of the galaxy. NGC 1581 is likely a companion of NGC 1566 (Kendall et al. 2015; Oh et al. 2015);see also Sect. 6).

NGC 1596/ NGC 1602. These two Dorado members, nearby in projection, are separated by $\Delta V_{\text {hel }}=230 \mathrm{~km} \mathrm{~s}^{-1}$ (Fig. C.10). NGC 1596 is a S0 galaxy (Type $=-2.0 \pm 0.5$ ). No H II regions are visible. NGC 1602 is an irregular galaxy (Type $=9.5 \pm 0.6)$. Our $\mathrm{H} \alpha+[\mathrm{N} \mathrm{II}]$ image outlines the presence of several $\mathrm{H}$ II regions which form a sort of ring and a large complex west of the galaxy centre.

\section{2. $H \alpha+[N$ II] equivalent width}

In Fig. 5 we plot the EW growth curve for each galaxy. Growth curves, computed following James et al. (2004), whose large sample is a benchmark reference, are obtained dividing the $\mathrm{H} \alpha+[\mathrm{N} \mathrm{II}]$ emission aperture fluxes by the continuum within the 


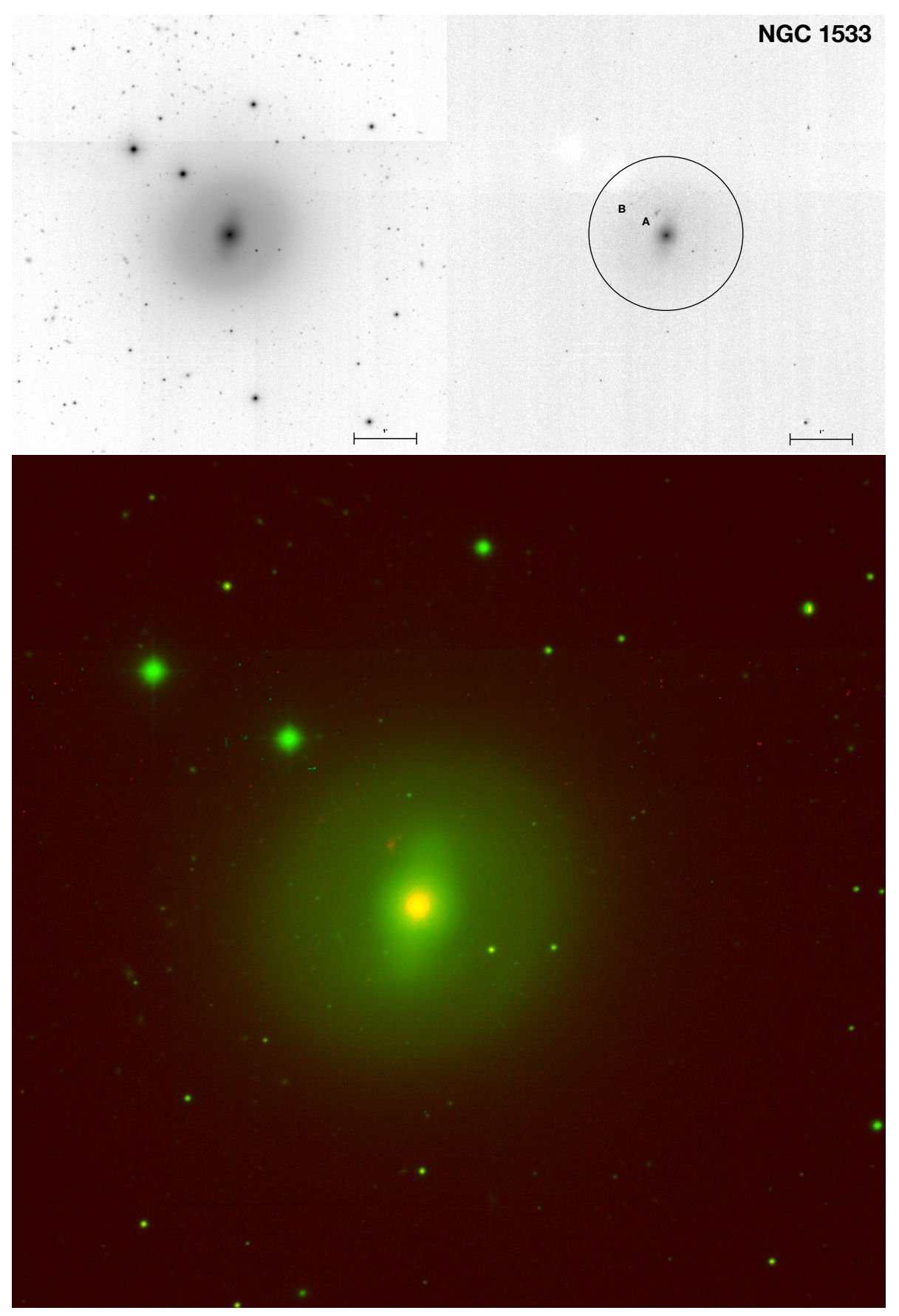

Fig. 4. As in Fig. 3 but for NGC 1533. Labels $\mathrm{A}$ and $\mathrm{B}$ indicate $\mathrm{H}$ II complexes (see text). The image size is $7^{\prime} \times 7^{\prime}$. same elliptical aperture, assuming that the average continuum level within the filter is equal to the continuum level in $\mathrm{H} \alpha \# 657$ filter. Total fluxes and EW are reported in Table 2. The EW of the $\mathrm{H} \alpha+[\mathrm{N} \mathrm{II}]$ emission traces the specific SFR, and is normalised by the luminosity of the older stellar population of the galaxy (see e.g. James et al. 2004; Lee et al. 2009).

Figure 5 shows that EW growth curves are basically flat in ETGs with average values of $\leq 0.75 \mathrm{~nm}$. NGC 1553, IC 2039, and NGC 1533 show higher EW values at the centre, namely 1,1 , and 1.5 , respectively. Moreover, the quasi-constant trend of NGC 1533 rests at a higher value than on average. NGC 1546 shows a rising inner curve reaching $E W=1.5$ between $10^{\prime \prime}$ and $20^{\prime \prime}$, which is in the bright ring, and then a slow decrease down to $E W=1$ in the outer region (about $\left.1^{\prime}\right)$. NGC 1581 shows almost the same trend as NGC 1546, reaching $E W=1.7$ at $12^{\prime \prime}$ and then slowly decreasing to
$E W=1$ at $35^{\prime \prime}$, which is the limit of emission we detect in this galaxy.

The growth curves for LTGs show different shapes. IC 2038 shows a rising curve out to $18^{\prime \prime}$ reaching a value of 2.5 units, before decreasing and stabilising around $E W=2$. In PGC 75125 the curve shows a plateau following the maximum value of $E W=2.5$ reached at 5". Growth curves of NGC 1536 and IC 2058 are decreasing from the centre to the periphery, while in NGC 1566, after reaching a minimum around $25^{\prime \prime}$, the curve shows a slight increase.

Concerning irregular galaxies, NGC 1602 shows the highest value in our sample of $E W=5.6$ at $30^{\prime \prime}$. In the outer region the curve decreases down to $E W=4.2$.

The total EW (Table 2) of our targets is plotted versus morphological type in Fig. 6. In the same figure we compare our results with those of James et al. (2004), who report 

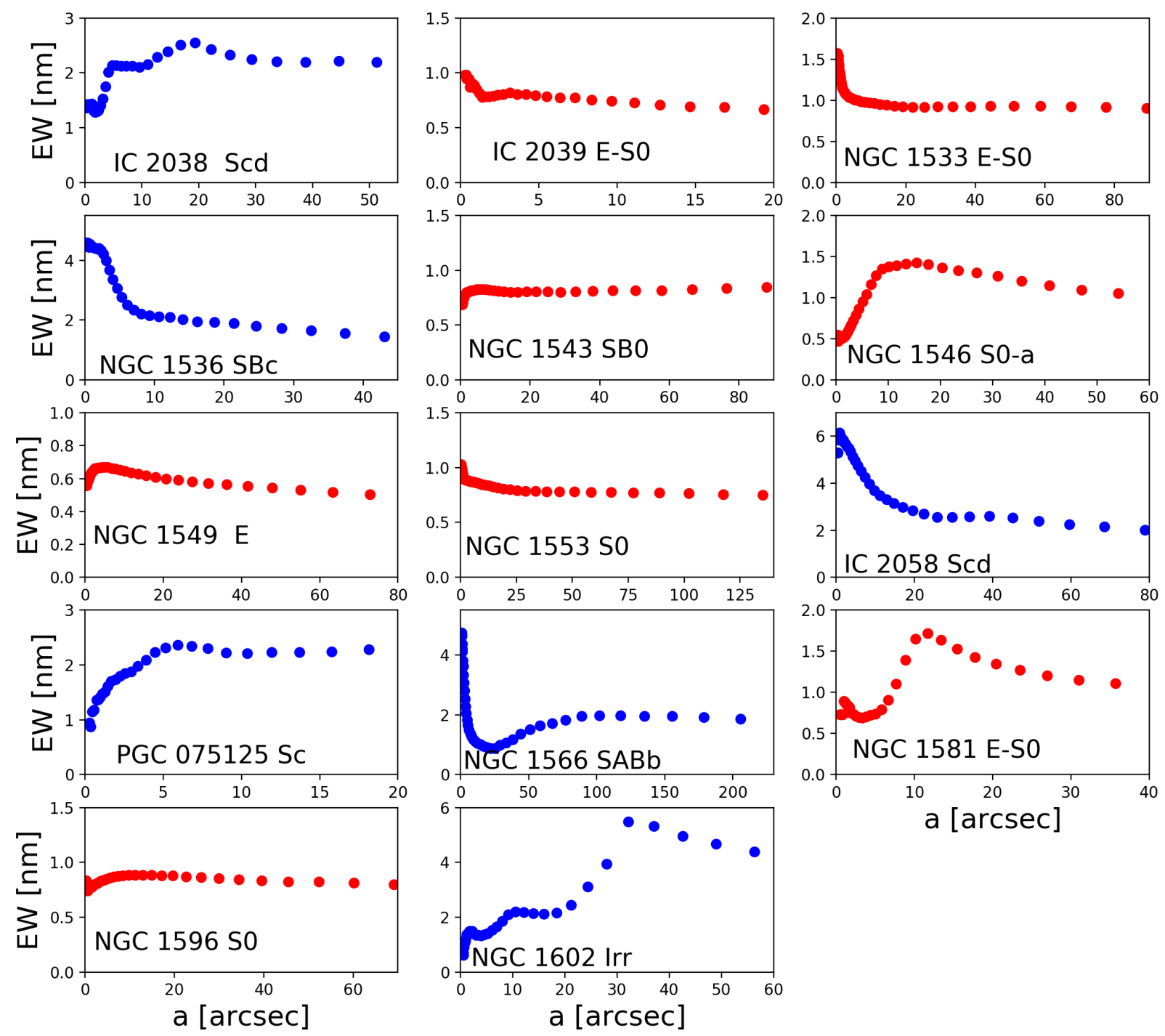

Fig. 5. $\mathrm{H} \alpha+[\mathrm{NII}]$ EW growth curve of each galaxy measured in concentric elliptical apertures out to the value of the elliptical aperture given in Table 2 and shown in Figs. 3 and 4 and in Appendix B (Figs. C.1-C.10). ETGs are indicated in red, LTGs in blue. Morphological classifications are from HyperLeda.

the mean EW as derived from a large sample of spiral galaxies, including interacting spirals as well as members of pairs and groups. These latter authors found an increasing trend of EW. Our results follow this trend although our targets with $4 \leq$ Type $\leq 8$ have values lower than the average and the median. However, spirals with values of $E W \approx 1.5-2.2$, as in our Dorado members, are present in the James et al. (2004) sample (see their Fig. 15). Although our sample is not large enough for our findings to be statistically significant, with respect to previous studies it extends the range of morphological types including several ETGs (the brigher ones) in the Dorado groups.

\subsection{Star formation rate}

The H $\alpha$ luminosities and SFRs are reported in the last columns of Table 3. Since our observations include [N II] we need to remove this contribution from the total flux. This implies knowledge of the $[\mathrm{N} \mathrm{III} / \mathrm{H} \alpha$ line ratio, for example from spectroscopy. An alternative is to use the average relationship between [N II] $\lambda 6583 / \mathrm{H} \alpha$ and the total B-band absolute magnitude $M_{B}$
(Col. 2 in Table 3), a consequence of the luminosity-metallicity relation for galaxies. We adopt the Lee et al. (2009) formulæ, in particular their equations 1 and 2, to derive the $[\mathrm{N} \mathrm{III}] / \mathrm{H} \alpha$ ratio, $\mathrm{L}(\mathrm{H} \alpha)$, and the SFR. Assuming a 3:1 ratio between $[\mathrm{N}$ II] $\lambda 6583$ and [N II] $\lambda 6548$, these latter authors propose the following formulæ. If $M_{B}>-20.3, \log ([\mathrm{N}$ III $] \lambda 6583 / \mathrm{H} \alpha)=$ $(-0.173 \pm 0.007) \times M_{B}-(3.903 \pm 0.137)$.

For $M_{B} \leq-20.3$, [N II] $] \lambda 6583 / \mathrm{H} \alpha=0.54$. For all LTGswith the exclusion of NGC 1566-, as they are fainter than -18 absolute $B$-mag, [N II] $/ \mathrm{H} \alpha \leq 0.16$ with an error of $<10 \%$. In this way, we compute the $\mathrm{H} \alpha$ luminosity, $\mathrm{L}(\mathrm{H} \alpha)$, in Table 3 and the SFR as: $\operatorname{SFR}\left(M_{\odot} \mathrm{yr}^{-1}\right)=7.9 \times 10^{-35} \mathrm{~L}(\mathrm{H} \alpha) \mathrm{W}$.

In Fig. 7 we plot the SFR as a function of morphological type. For Type $>0$, three of our targets are consistent with the trend reported by James et al. (2004, for a sample of 334 spiral galaxies, of morphological types from S0/a to Irr and with $V_{\text {hel }} \leq$ $3000 \mathrm{~km} \mathrm{~s}^{-1}$ ), increasing until Type $\sim 4-5$ then declining, while another three targets, PGC 75125, IC 2038, and NGC 1536, have significantly lower SFR, comparable with Type $<0$ sample galaxies. We point out that LTGs with low SFR, like the cases above, are also found in the James et al. (2004) sample. PGC 75125, in 


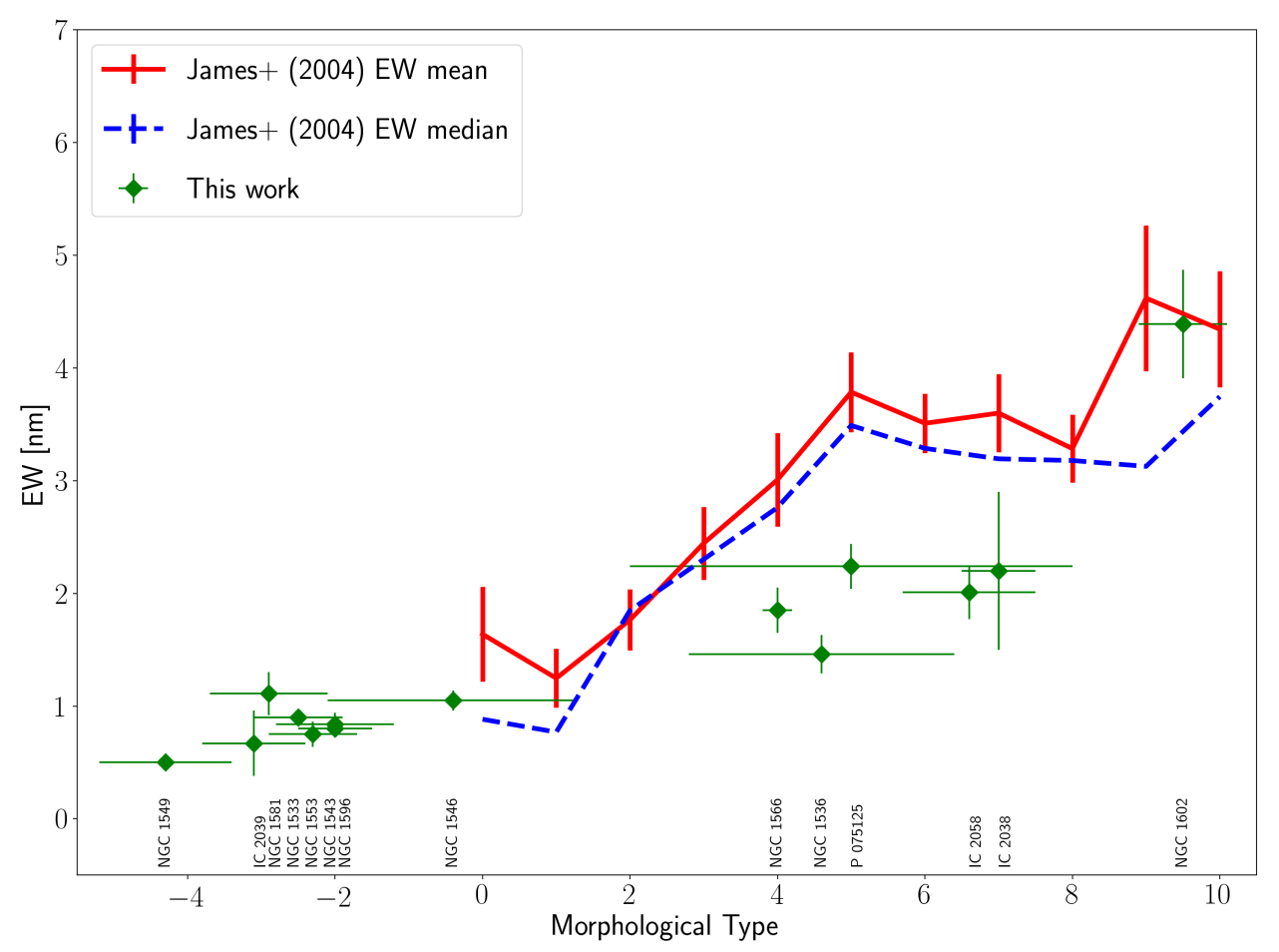

Fig. 6. Integrated value of $\mathrm{H} \alpha+[\mathrm{NII}] \mathrm{EW}$ for our targets as a function of their morphological type. Our values are compared with the mean and the median for the latetype galaxy sample studied by James et al. (2004).
Table 3. Parameters used to compute the SFR from $\mathrm{H} \alpha$ luminosity.

\begin{tabular}{lcccc}
\hline \hline $\begin{array}{l}\text { ID } \\
\text { source }\end{array}$ & $M_{B}$ & $E(B-V)$ & $\begin{array}{c}L(\mathrm{H} \alpha) \\
{\left[10^{33} \mathrm{~W}\right]}\end{array}$ & $\begin{array}{c}\mathrm{SFR}_{\mathrm{H} \alpha} \\
{\left[M_{\odot} \mathrm{yr}^{-1}\right]}\end{array}$ \\
\hline IC 2038 & -15.87 & 0.010 & $0.4 \pm 0.1$ & $0.03 \pm 0.01$ \\
IC 2039 & -16.30 & 0.010 & $0.009 \pm 0.004$ & $0.008 \pm 0.003$ \\
NGC 1533 & -19.52 & 0.016 & $4.9 \pm 0.2$ & $0.40 \pm 0.02$ \\
NGC 1536 & -17.80 & 0.019 & $1.42 \pm 0.05$ & $0.118 \pm 0.004$ \\
NGC 1543 & -19.88 & 0.024 & $4.4 \pm 0.5$ & $0.37 \pm 0.04$ \\
NGC 1546 & -19.24 & 0.013 & $2.6 \pm 0.1$ & $0.21 \pm 0.09$ \\
NGC 1549 & -20.61 & 0.011 & $4.2 \pm 0.2$ & $0.4 \pm 0.02$ \\
NGC 1553 & -21.02 & 0.013 & $11.9 \pm 1.4$ & $1.0 \pm 0.1$ \\
IC 2058 & -17.55 & 0.014 & $1.1 \pm 0.1$ & $1.01 \pm 0.01$ \\
PGC 75125 & -15.81 & 0.014 & $0.11 \pm 0.01$ & $0.01 \pm 0.01$ \\
NGC 1566 & -20.99 & 0.008 & $27.2 \pm 2.7$ & $2.2 \pm 0.2$ \\
NGC 1581 & -17.71 & 0.007 & $1.6 \pm 0.1$ & $0.13 \pm 0.01$ \\
NGC 1596 & -19.27 & 0.008 & $3.3 \pm 0.2$ & $0.27 \pm 0.02$ \\
NGC 1602 & -17.87 & 0.009 & $3.0 \pm 0.2$ & $0.25 \pm 0.02$ \\
\hline
\end{tabular}

Notes. Parameters used to compute the $\mathrm{H} \alpha$ fluxes and the galaxy SFR Column 1 provide source identity. Column 2 gives the absolute $B$-band magnitude (17.69 Mpc from Kourkchi \& Tully 2017). Column 3 is the $E(B-V)$ extinction. Column 4 and Col. 5 report the $\mathrm{H} \alpha$ luminosity and the SFR ${ }_{H \alpha}$. The SFR has been computed from the $\mathrm{H} \alpha$ luminosity according to Lee et al. (2009) (see text in Sect. 5.3).

particular, has a very uncertain classification and is likely near to S0s (see our Sect. 5).

Dorado is not sufficiently rich in spirals to define a trend for Types $>0$, but its galaxy population includes several early types. In Fig. 7 we compare our results with the SFR calculated by Gavazzi et al. (2018) from $\mathrm{H} \alpha+[\mathrm{N}$ II] imaging of a sample of 147 ETGs from ATLAS ${ }^{3 \mathrm{D}}$. These latter authors detect 55 EGTs (37\% of the sample), mostly S0. To compute the SFR, Gavazzi et al. (2018) adopted the Kennicutt (1998) formula modified by a Chabrier IMF. As $\operatorname{SFR}($ Chabrier $)=\operatorname{SFR}($ Kennicutt $) / 1.58$ (Boselli et al. 2015), we applied this transformation to the sample in Gavazzi et al. (2018) to compare their values with our estimates.

Gavazzi et al. (2018) divided ETGs into two sets: ellipticals (Type $=-5$, magenta squares) and S0s (Type $=-2$, cyan squares). Four of our galaxies show a larger SFR than those found by Gavazzi et al. (2018), namely three S0s (NGC 1533, NGC 1543, NGC1553) and NGC 1549, a bona fide elliptical. NGC 1553 has a SFR that, remarkably, is above the average for ETGs, despite its EW value. Figure 8 (adapted from Rampazzo et al. 2003), discussed in Sect. 6.1, provides a clear map of the presence and complex structure of the $\mathrm{H} \alpha$ emission in the inner region of this galaxy.

\section{Discussion}

It is widely accepted that cluster galaxies have depressed SFRs in comparison with the field (e.g. Poggianti et al. 2006; Vulcani et al. 2010; Paccagnella et al. 2016, and references therein). Understanding SF quenching during the galaxy coevolution in groups is fundamental for two reasons: groups contain $\approx 60 \%$ of the galaxies in the nearby universe (Tully \& Fisher 1988; Ramella et al. 2002; Tago et al. 2008) and the transition in galaxy properties that is typical between field galaxies and clusters takes place roughly at the characteristic densities of groups (Lewis et al. 2002; Goto et al. 2003; Gómez et al. 2003; Marino et al. 2016). In the following, we discuss our results considering substructures in the Dorado group, from its barycentre to the periphery.

\subsection{The Dorado barycentre: the compact group SCG 0414-5559}

The barycentre of Dorado hosts the compact group SGC 04145559 identified by Iovino (2002) (for the compact group definition see Hickson 1982). SGC 0414-5559 includes four members, namely NGC 1546 (member C), NGC 1549 (member B), NGC 1553 (member A), and IC 2058 (member D). The 


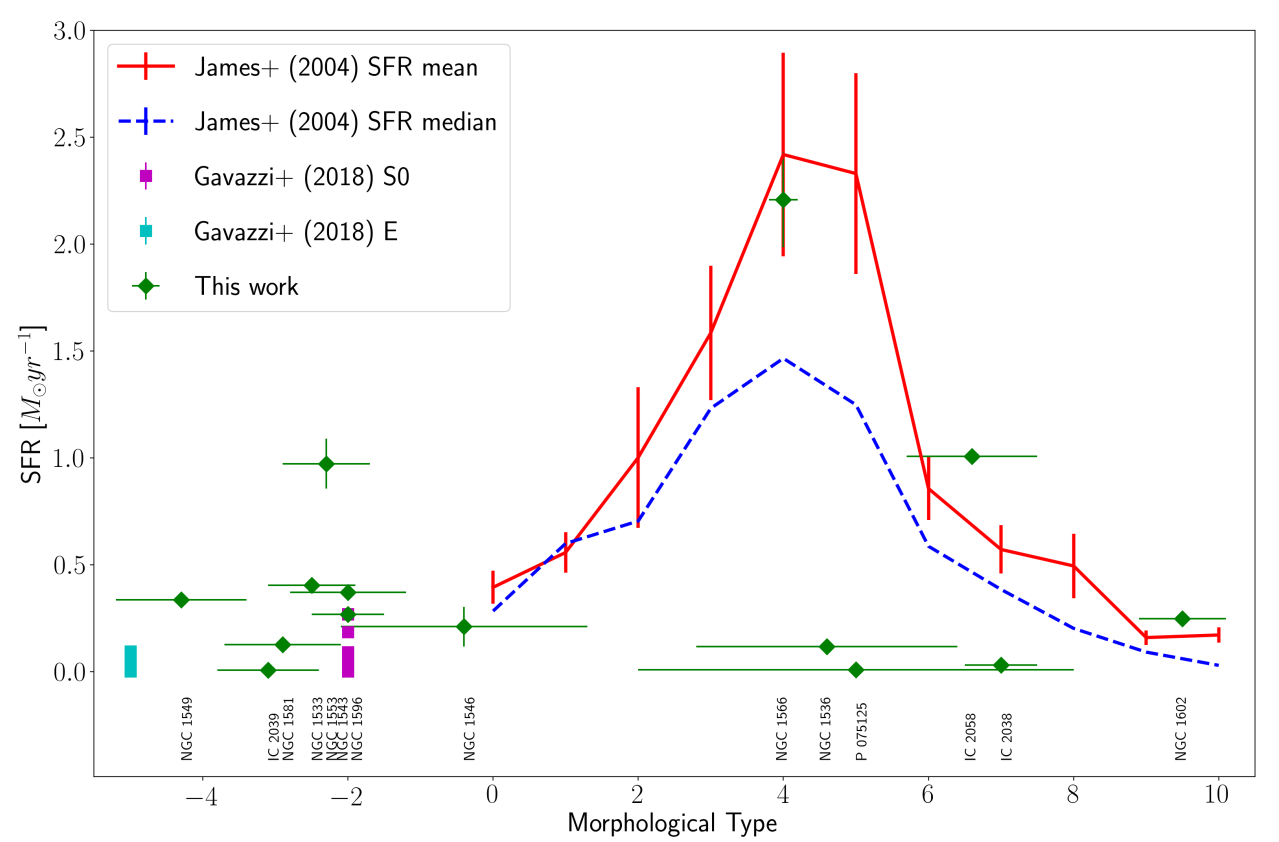

Fig. 7. $\mathrm{H} \alpha$ SFR of Dorado galaxies as a function of their morphological type. For LTGs $(0 \leq$ Type $\leq 10)$ the red line and the blue dotted lines represent the mean and the median SFR computed on a sample of 334 galaxies by James et al. (2004). For ETGs $(-5 \leq$ Type $<0)$ the magenta and cyan squares represent the SFR of detected objects (55/147), representative of the whole ATLAS ${ }^{3 \mathrm{D}}$ survey, by Gavazzi et al. (2018). These latter authors divided ETGs into two families of elliptical (Type $=-5$ ) and S0s (Type $=-2$ ). Our values (green diamonds) are reported in Table 3.

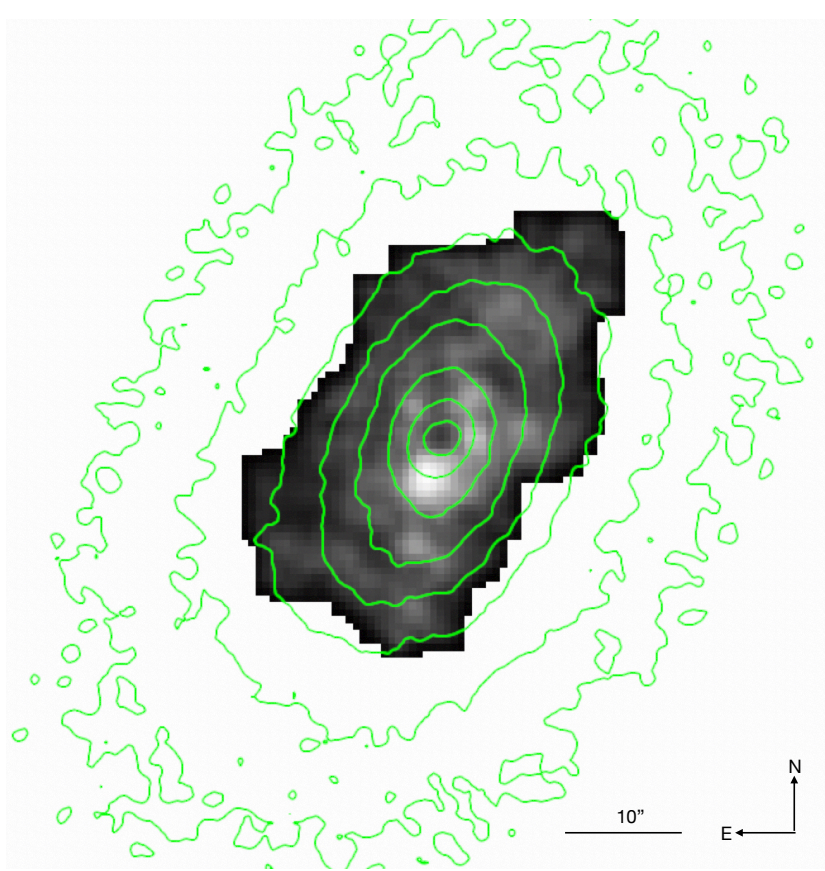

Fig. 8. NGC 1553: H $\alpha$ monochromatic map obtained from Fabry-Perot (Rampazzo et al. 2003) overplotted to the present $\mathrm{H} \alpha+[\mathrm{N} \mathrm{II}]$ contours. The $\mathrm{H} \alpha$ emission is clumpy and extends for about $30^{\prime \prime}$. We note that the $\mathrm{H} \alpha$ peak is displaced from the $\mathrm{H} \alpha+[\mathrm{N} \mathrm{II}]$ centre and corresponds to the X-ray source Chandra CXOU J041610.5-554646.8.

average recession velocity of the four galaxies is $1259 \mathrm{~km} \mathrm{~s}^{-1}$ with a standard deviation of $93 \mathrm{~km} \mathrm{~s}^{-1}$ (see Fig. A.1 and Table A.1).

Diaferio et al. (1994) proposed that compact groups form continually in a single rich group during its collapse and virialisation. This idea is based on observational evidence that compact groups are located within looser structures or rich neighbourhoods (see e.g. Ribeiro et al. 1998, and references therein). According to the $N$-body simulations of Diaferio et al.
(1994), the survival time of these structures is about $1 \mathrm{Gyr}$ and most of the member galaxies are not merger remnants.

As sketched in Sect. 2, galaxies in SCG 0414-5559 are, in contract, rich in merging and interaction signatures. The nuclei of the two shell galaxies, NGC 1549 and NGC 1553, are LINERs (see e.g. Annibali et al. 2010; Rampazzo et al. 2013). Optical line strength indices show that NGC 1553 has a young nucleus with a luminosity-weighted age of $4 . \pm 0.7 \mathrm{Gyr}$ (Annibali et al. 2007). Mid-infrared Spitzer-IRS spectra of both NGC 1549 and NGC 1553 from Rampazzo et al. (2013) show that PAHs are present in the nucleus with anomalous emission ratios. This kind of nucleus could host AGNs as well as residuals of past star formation events (see e.g. Vega et al. 2010). In the X-ray domain, the emission of the nucleus of NGC 1553 is AGNlike (Flohic et al. 2006). GALEX observations detected both NGC 1553 (Marino et al. 2011b) and NGC 1549 in NUV while a ring is barely visible in FUV in NGC 1553.

Figures C. 4 and C. 5 show that the $\mathrm{H} \alpha+[\mathrm{N} \mathrm{II]}$ emission distribution, along the galaxy body, is following the stellar continuum. Gavazzi et al. (2018) suggest that weak $\mathrm{H} \alpha$ emitters, for example galaxies dominated by [N II], can harbours AGN at their centre, not connected with the observed widespread SF. Spectroscopic studies illustrate and enrich the above finding. Rampazzo et al. (2003) used Fabry-Perot observations to study the $\mathrm{H} \alpha$ kinematics in the central regions of NGC 1553. The high spectral resolution $(R=9400$ at $\mathrm{H} \alpha$, for $S / N=3)$ of the instrument made it possible to isolate $\mathrm{H} \alpha$ emission showing that gas and stars co-rotate. In Fig. 8 we overplot the $\mathrm{H} \alpha$ monochromatic map from the Fabry-Perot to our $\mathrm{H} \alpha+[\mathrm{N}$ II] isophotal contours. The map shows the clumpy and irregular $\mathrm{H} \alpha$ distribution, whose peak is centred neither on the continuum nor on the $\mathrm{H} \alpha+[\mathrm{N} \mathrm{II}]$ emission but coincides with an X-ray discrete source whose colours suggest an absorbed AGN (Blanton et al. 2001; Flohic et al. 2006). The X-ray-discrete source, Chandra CXOU J041610.5-554646.8, seems partly responsible for the very innermost $\mathrm{H} \alpha$ emission of NGC 1553.

The outskirts of NGC 1553 are characterised by very extended shells (Malin \& Carter 1983). We searched for extragalactic HII regions and dwarf galaxies in our FOV. [CMI2001]4136-0 is an object that is highly visible in GALEX 

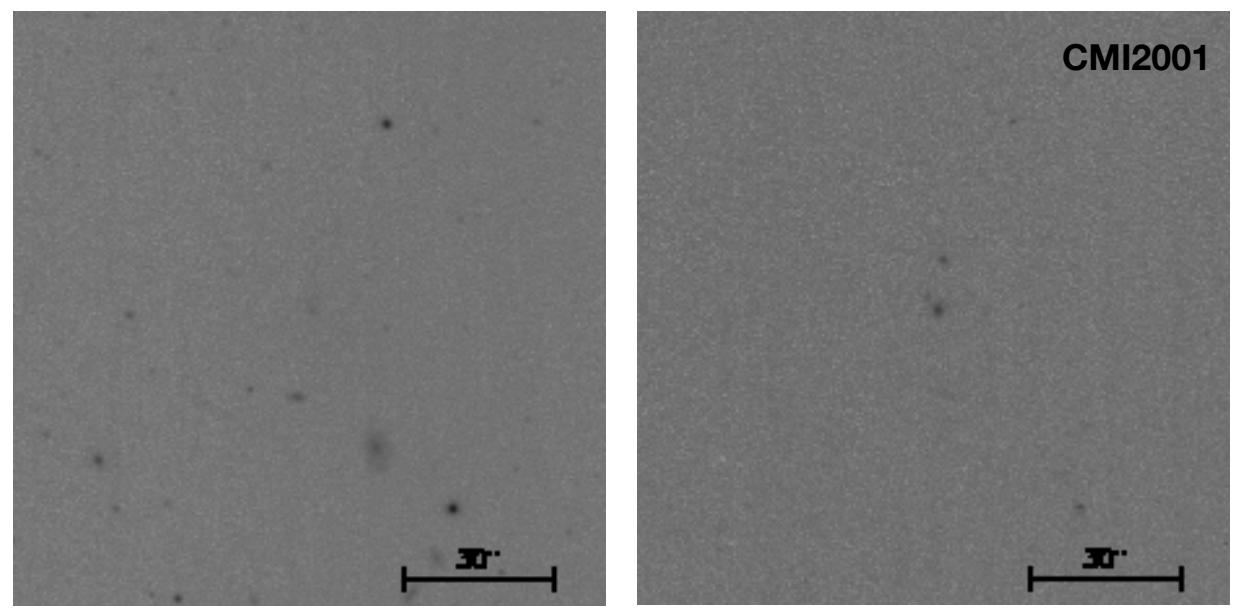

Fig. 9. [CMI2001]4136-01 (RA = 0416 15.1 Dec $=-554151 \mathrm{~J} 2000)$ is a region northeast of NGC 1553 that has been detected by GALEX. A zoom on this area is shown in the continuum (left panel) and in $\mathrm{H} \alpha+[\mathrm{N} \mathrm{II}]$ (right panel). The FOV is $2^{\prime} \times 2^{\prime}$. In the centre of the field, two H II regions are clearly visible in $\mathrm{H} \alpha+[\mathrm{N}$ II] frame, while objects are barely detected in the continuum.

FUV projected on the NGC 1553 outskirts (Fig. 9). Although it is classified as a galaxy in CDS, we we do not see any obvious galaxy in continuum frame. Rather, in $\mathrm{H} \alpha$ we see two H II regions which should be associated to NGC 1553.

The region of IC 2058, southeast of NGC 1549/NGC 1553, is $\mathrm{H} \mathrm{I}$-rich (Kilborn et al. 2009) with a mass of $17.1 \pm 1.2 \times 10^{8} M_{\odot}$ Pearson et al. (2016) studied the H I distribution enclosing IC 2058 and the dwarf galaxy PGC 75125. On the basis of the low outer versus total H I gas fraction, these latter authors suggest that most of the HI gas, removed in the IC2058/PGC 75125 galaxy-galaxy interaction, is in an ionised state. Both IC 2058 and PGC 75125 show H II complexes (see also Rossa \& Dettmar 2003, for IC2058). The $\mathrm{H} \alpha+[\mathrm{N} \mathrm{II}]$ emission, crossing PGC 75125 as a clumpy and warped lane, may suggest an in-flight gas refueling (see e.g. Domingue et al. 2003, and references therein) following the picture described by Pearson et al. (2016).

NGC 1546 is located southwest of NGC 1549 and NGC 1553. Kilborn et al. (2005) detected this galaxy in $\mathrm{HI}$ with a mass of $24.1 \pm 1.4 \times 10^{8} M_{\odot}$. Its $\mathrm{H}$ II regions have a flocculent appearance likely following the inner and outer rings described by Comerón et al. (2014).

In summary, the SF is concentrated at the southern periphery of the SCG 0414-5559 compact group in correspondence with a rich concentration of $\mathrm{HI}$, while the area covered by NGC 1549 and NGC 1553 is undetected down to the Kilborn et al. (2005) survey limit of $\approx 3.5 \times 10^{8} M_{\odot}$. However, $\mathrm{H} \alpha$ was detected using Fabry-Perot in the central 30" by Rampazzo et al. (2003) and in the present study as H II regions in the NGC 1553 outskirts.

\subsection{The northwest side of the Dorado backbone: NGC 1533, IC 2038, and IC 2039}

The northwest side of Dorado is a physical substructure in the group (see e.g. Fig. A.1 bottom panel). Ryan-Weber et al. (2003) detected a wide plume of H I extending from IC 2038 to the east towards NGC 1533. Several extragalactic H II regions were detected in this H I tail by Werk et al. (2010, their Fig. 7).

Figure 4 shows that SF in NGC 1553 is not shut down: H II regions, indicated with $\mathrm{A}$ and $\mathrm{B}$, are outside the galaxy centre in a SF ring shown by Marino et al. (2011a) in NUV and FUV. We searched for H II regions detected by Werk et al. (2010). The H II regions shown in Fig. 10 correspond to the Werk et al. (2010) detection. However, the triplet merits further investigation with wide-field, deep $\mathrm{H} \alpha+[\mathrm{N} \mathrm{II}]$ images (see e.g. Boselli et al. 2018).

Our observations confirm that SF is detected not only in the spiral galaxy IC 2038 and in the centre of NGC 1533 but also along the H I tails. The SF in IC 2038 is depressed if compared with objects of the same morphological type (Fig. 7), likely because of ongoing stripping (see e.g. Werk et al. 2010; Cattapan et al. 2019).

\subsection{The southwest edge of Dorado: NGC 1536 and NGC 1543}

NGC 1536 and NGC 1543 appear quite isolated in projection within the group, although their heliocentric velocities $\left(V_{\text {hel }}=\right.$ 1296 and $V_{\text {hel }}=1148 \mathrm{~km} \mathrm{~s}^{-1}$, respectively) are very close to that of the central pair, NGC 1549 and NGC $1553\left(V_{\text {hel }}=\right.$ $1202 \mathrm{~km} \mathrm{~s}^{-1}$ ). The structure of NGC 1536 is relatively asymmetric. Both NGC 1536 and NGC 1543 were detected in $\mathrm{H} \alpha+[\mathrm{N}$ II] $\mathrm{H}$ II regions are revealed throughout the body of NGC 1536 but the galaxy has a very low SFR (Fig. 7). Kilborn et al. (2005) noted that $\mathrm{HI}$ is detected at a rate that is five to ten times less than expected for this galaxy.

$\mathrm{H}$ II regions are detected in the outer ring in NGC 1543. In the central 1.5" of NGC 1543, Parkash et al. (2019) measured a ratio of $[\mathrm{NII}] / \mathrm{H} \alpha=2.004$, confirming that, as in the case of NGC 1553, the dominant phenomenon is not SF, at least in the nucleus. Indeed, the galaxy nucleus is classified as a LINER. Although NGC 1543 is classified as a barred S0 (see Table A.1), Murugeshan et al. (2019, and references therein) included the galaxy in a sample of H I -deficient late-type galaxies (see also Kilborn et al. 2005). Investigating the possible role of the angular momentum in the $\mathrm{HI}$ depletion $\left(\log _{\mathrm{HI}}=8.75 M_{\odot}\right)$, these latter authors show that $\mathrm{HI}_{\mathrm{I}}$ is concentrated in the galaxy outskirts, overlapping the stellar outer ring. The galaxy main body is devoid of HI at the limit of their survey. The H II complexes we find in this galaxy develop at the southeast edge of the H I and stellar ring. Murugeshan et al. (2019) suggested that the HI hole in NGC 1543 is regulated by the galaxy specific angular momentum rather than the influence of the environment.

Marino et al. (2011a) found FUV emission in the same area in which we found H II regions. Mazzei et al. (2019) showed that the global properties of this galaxy, both photometric and spectro-photometric (SED, B absolute magnitude, morphology, velocity dispersion and rotation, X-ray luminosity of the hot gas, and amount of H I gas) can be accounted for by a minor merger event (mass ratio 5:1). The galaxy has a global age of 10.7 Gyr and suffered rejuvenation episodes in the last $2.3 \mathrm{Gyr}$. In these galaxies, the "H I deficiency" seems dominant in regulating the SF. 


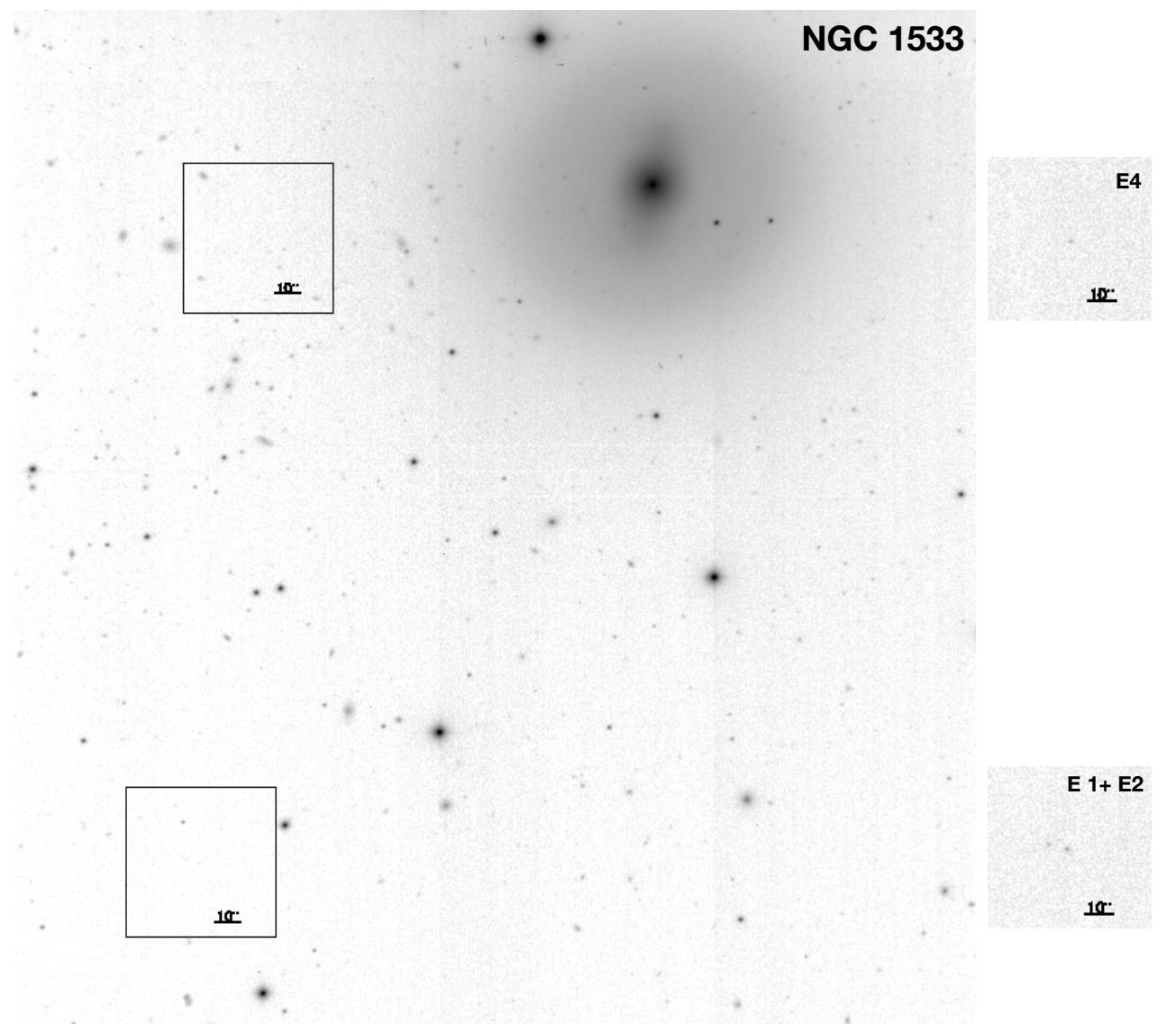

Fig. 10. Left panel: southeast region of NGC 1533 shown in the continuum nearby $\mathrm{H} \alpha$. The two areas of $60^{\prime \prime} \times 60^{\prime \prime}$ inserted in the panel mark the position of $\mathrm{H}$ II regions revealed by our $\mathrm{H} \alpha+[\mathrm{N}$ II] observations, E1+ E2 (04 1013.5 -56 $1136 \mathrm{~J} 2000)$ and E4 (04 1010.66 $-560727.99 \mathrm{~J} 2000)$ and shown in the two right panels. These $\mathrm{H}$ II regions were first identified by Werk et al. (2010).

\subsection{Towards the east along the backbone: the pairs NGC 1566/NGC 1581 and NGC 1596/NGC 1602}

Three objects are found in projection east of the SCG 04145559 central compact group along the Dorado backbone, namely NGC 1566, NGC 1581, and the clump formed by NGC 1596 and NGC 1602. There is evidence that not only the two close galaxies NGC 1596 and NGC 1602 form a physical pair but also the very separated ones NGC 1566 and NGC 1581. Both regions are H I rich (Kilborn et al. 2005; Chung et al. 2006).

Kendall et al. (2015) and Oh et al. (2015) suggest that NGC 1566 and NGC 1581 form a physical pair of galaxies, although their projected separation in terms of galaxy size, $R_{\text {proj }} / R_{25}$, is $9.7 \mathrm{kpc}$. This pair, with a relative mass ratio of 0.05 and a radial velocity difference of $96 \mathrm{~km} \mathrm{~s}^{-1}$, has a high tidal parameter (a measure of the strength of the tidal interaction) according to Kendall et al. (2015). The arm structures of NGC 1566 (Fig. C.8) still show signatures of an encounter that might have occurred with NGC 1581 (Oh et al. 2015), while NGC 1581 does not show obvious signatures of interaction (see Fig. C.9). However, NGC 1581, which shows H II regions in the inner ring, could have acquired the gas from NGC 1566 during its peri-galactic passage. Several acquisition and gas-stripping scenarios have been discussed in the case of mixed ETG+spiral pairs by Domingue et al. (2003), while mass-transfer examples are in described in detail by Keel (2004).

The case of the physical pair NGC 1596/NGC 1602 (Fig. C.10) may be another example of H I gas transfer by a donor. The area in which the pair is found is very rich in neutral hydrogen (Chung et al. 2006) which extends from NGC 1602, the donor, to NGC 1596, the receiver. In NGC 1596, the acquired gas counter-rotates with respect to stars. However, we do not detect H II regions in NGC 1596 but only an extended
$\mathrm{H} \alpha+[\mathrm{N} \mathrm{II}]$ emission. Chung et al. (2006) reported that the ionised gas in NGC 1596 produces mostly [OIII] $\lambda 5007$ emission in the central part (see also Bureau \& Chung 2006). There is no mention of $\mathrm{H} \alpha$ by Bureau \& Chung (2006), although one channel (of the dual-channel spectrograph) is centred on the $\mathrm{H} \alpha$ wavelength. The [OIII] emission is concentrated in the central regions (of a few arcsec; see Fig. 1 in Bureau \& Chung 2006).

\section{Summary and conclusions}

Dorado is a nearby, rich group extending for about 10 square degrees in the southern hemisphere. We observed the 14 galaxies that form the group backbone, both early and late types, in narrow-band $\mathrm{H} \alpha+[\mathrm{N} \mathrm{II}]$ imaging. We obtained their $\mathrm{H} \alpha$ luminosity and estimated their SFR.

We obtained the following results:

- All members were detected in $\mathrm{H} \alpha+[\mathrm{N} \mathrm{II}]$, irrespective of their morphological type.

- H II regions are clearly visible in half of the ETGs, namely NGC 1533, NGC 1543, NGC 1546, and NGC 1581. H II regions are found in rings and/or filaments. In NGC 1549, NGC 1553, and NGC 1596, the $\mathrm{H} \alpha+[\mathrm{N} \mathrm{II}]$ emission does not show the clumpy structures of H II regions, but a rather smooth structure. However, $\mathrm{H} \alpha$ is likely present in the central regions of the galaxy as suggested by the high-resolution Fabry-Perot observations of NGC 1553 (Rampazzo et al. 2003).

- We detect H II regions in the galaxy outskirts of NGC 1533 (Werk et al. 2010, and reference therein) and in NGC 1553, detected also by GALEX as FUV-emitting regions.

- Gavazzi et al. (2018), using the ATLAS ${ }^{3 \mathrm{D}}$ sample, detected $55 / 147$ ETGs, which is $37 \%_{-11}^{+6}$ of their sample (errors 
are calculated using $1 \sigma$ low and upper limits from Poisson statistics following Gehrels 1986). Considering errors, Gavazzi et al. (2018) detected less than half of their galaxies in $\mathrm{H} \alpha+[\mathrm{N} \mathrm{II}]$, likely because of the significant presence of Virgo members which include passively evolving ETGs (see e.g. Bressan et al. 2006). We conclude that ETGs in Dorado are leaving an active phase and their SF is not yet extinguished.

- The HII regions of the LTG NGC 1536 are irregularly distributed following the morphological perturbation of the underlying galaxy. This is also partially seen in IC 2038 .

- The EW of $\mathrm{H} \alpha+[\mathrm{N}$ II] emission, a measure of the specific SFR, is increasing with morphological type, although most of the LTGs in our sample are below the mean and median values corresponding to their morphological types (James et al. 2004). The EW of ETGs extends the trend towards low morphological-type values, in the $-5 \leq$ Type $\leq 0$ range.

- The SFR of the Dorado spiral members is in the range of general surveys of LTGs (James et al. 2004), but rarely above the median of their morphological type. Three galaxies, namely NGC 1536, PGC 75125, and IC 2058, have a SFR that is well below the median for their morphological classes.

The dominant mechanisms in action in this evolutionary phase of the Dorado group are gas stripping and gas exchange via in-flight re-fuelling between galaxies. Some galaxies are already $\mathrm{H}$ I deficient, as in cluster counterparts. In summary, the present $\mathrm{H} \alpha+[\mathrm{N}$ II] observations show the Dorado backbone to be a strongly evolving environment. $\mathrm{H} \alpha$ is a short-timescale $\left(10^{7}\right.$ years) indicator in terms of evolution. The gas reservoirs, still present in LTGs and their surrounding environment, sometimes trigger a residual activity in ETGs.

The donor-receiver mechanism, via galaxy-galaxy interaction, appears important at the edge of Dorado, considering the pairs (NGC 1566/ NGC 1581, NGC 1596/ NGC 1602) and the triplet (NGC 1533, IC 2038, and IC 2039). Gas stripping is another mechanisms in action: NGC 1533 stripped IC 2038, and the same might have happened in the past between NGC 1566 and NGC1581 and between NGC 1596 and NGC 1602. The consequence of this stripping could be linked to the low SFR of the spiral IC 2038 (Fig. 7), to the presence of H II regions in the inner ring of NGC 1581, and to the H I plume connecting NGC 1602 to NGC 1596 (Chung et al. 2006).

The barycentre of the group, the compact group SCG 04145559, appears more evolved. In the two shell galaxies NGC 1549 and NGC 1553, the $\mathrm{H} \alpha+[\mathrm{N} \mathrm{II}]$ emission is dominated by [NII], although $\mathrm{H} \alpha$ has been found in the NGC 1553 central region via Fabry-Perot high-resolution observations (Rampazzo et al. 2003). Star formation is still found in the outskirts of this compact group in IC 2058, in its dwarf physical companion PGC 75125, both enclosed in an H I cloud, and in NGC 1546.

Analysis of the SFR in Dorado will continue in the farultraviolet. We have already observed the present sample with UVIT on Astrosat (Tandon et al. 2017) in the FUV band. The connection between UV and $\mathrm{H} \alpha$ SFRs will be the subject of a forthcoming paper.

Acknowledgements. We wish to thank the unknown referee for very constructive suggestions. R.R. and P.M acknowledge the partial support of the INAF PRIN-SKA 2017 program 1.05.01.88.04. MS acknowledges financial support from the VST project (P.I. P. Schipani). E.C. acknowledges support from ANID project Basal AFB-170002. This paper includes data gathered with the du Pont and Swope Telescopes located at Las Campanas Observatory, Chile. We acknowledge the usage of the HyperLeda database (http://leda.univ-lyon1.fr). IRAF is distributed by the National Optical
Astronomy Observatories, which is operated by the Association of Universities for Research in Astronomy, Inc. (AURA) under cooperative agreement with the National Science Foundation.

\section{References}

Annibali, F., Bressan, A., Rampazzo, R., Zeilinger, W. W., \& Danese, L. 2007, A\&A, 463, 455

Annibali, F., Bressan, A., Rampazzo, R., et al. 2010, A\&A, 519, A40

Arp, H. C., \& Madore, B. 1987, A catalogue of Southern Peculiar Galaxies and Associations

Baldry, I. K., Glazebrook, K., Brinkmann, J., et al. 2004, ApJ, 600, 681

Balogh, M. L., Baldry, I. K., Nichol, R., et al. 2004, ApJ, 615, L101

Barnes, J. E. 2002, MNRAS, 333, 481

Bertin, E., Mellier, Y., Radovich, M., et al. 2002, in Astronomical Data Analysis Software and Systems XI, eds. D. A. Bohlender, D. Durand, T. H. Handley, ASP Conf. Ser., 281, 228

Blanton, E. L., Sarazin, C. L., \& Irwin, J. A. 2001, ApJ, 552, 106

Boselli, A., Fossati, M., Gavazzi, G., et al. 2015, A\&A, 579, A102

Boselli, A., Fossati, M., Ferrarese, L., et al. 2018, A\&A, 614, A56

Boselli, A., \& Gavazzi, G. 2006, PASP, 118, 517

Boselli, A., \& Gavazzi, G. 2014, A\&ARv., 22, 74

Bressan, A., Panuzzo, P., Buson, L., et al. 2006, ApJ, 639, L55

Brough, S., Forbes, D. A., Kilborn, V. A., \& Couch, W. 2006, MNRAS, 370, 1223

Bureau, M., \& Chung, A. 2006, MNRAS, 366, 182

Carrasco, E. R., Mendes de Oliveira, C., Infante, L., \& Bolte, M. 2001, AJ, 121, 148

Cattapan, A., Spavone, M., Iodice, E., et al. 2019, ApJ, 874, 130

Chung, A., Koribalski, B., Bureau, M., \& van Gorkom, J. H. 2006, MNRAS, 370,1565

Comerón, S., Salo, H., Laurikainen, E., et al. 2014, A\&A, 562, A121

Di Matteo, T. 2015, in IAU Gen. Assembly, 29, 2257908

Diaferio, A., Geller, M. J., \& Ramella, M. 1994, AJ, 107, 868

Domingue, D. L., Sulentic, J. W., Xu, C., et al. 2003, AJ, 125, 555

Dressler, A., \& Shectman, S. A. 1988, AJ, 95, 985

Dupraz, C., \& Combes, F. 1987, A\&A, 185, L1

Ferguson, H. C., \& Sandage, A. 1990, AJ, 100, 1

Firth, P., Evstigneeva, E. A., Jones, J. B., et al. 2006, MNRAS, 372, 1856

Flohic, H. M. L. G., Eracleous, M., Chartas, G., Shields, J. C., \& Moran, E. C. 2006, ApJ, 647, 140

Garcia, A. M. 1993, A\&AS, 100, 47

Gavazzi, G., Consolandi, G., Pedraglio, S., et al. 2018, A\&A, 611, A28

Gehrels, N. 1986, ApJ, 303, 336

Gómez, P. L., Nichol, R. C., Miller, C. J., et al. 2003, ApJ, 584, 210

Goto, T., Yamauchi, C., Fujita, Y., et al. 2003, MNRAS, 346, 601

Goudfrooij, P., Hansen, L., Jorgensen, H. E., \& Norgaard-Nielsen, H. U. 1994a, A\&AS, 105, 341

Goudfrooij, P., Hansen, L., Jorgensen, H. E., et al. 1994b, A\&AS, 104, 179

Hawley, S. A., \& Phillips, M. M. 1980, ApJ, 235, 783

Heckman, T. M. 1980, A\&A, 500, 187

Hickson, P. 1982, ApJ, 255, 382

Hoopes, C. G., Walterbos, R. A. M., \& Bothun, G. D. 2001, ApJ, 559, 878

Huchra, J. P., \& Geller, M. J. 1982, ApJ, 257, 423

Iovino, A. 2002, AJ, 124, 2471

James, P. A., Shane, N. S., Beckman, J. E., et al. 2004, A\&A, 414, 23

Jeong, H., Yi, S. K., Bureau, M., et al. 2009, MNRAS, 398, 2028

Kaviraj, S., Schawinski, K., Devriendt, J. E. G., et al. 2007, ApJS, 173, 619

Keel, W. C. 2004, AJ, 127, 1325

Kendall, S., Clarke, C., \& Kennicutt, R. C. 2015, MNRAS, 446, 4155

Kennicutt, R. C., Jr. 1998, ARA\&A, 36, 189

Kennicutt, R. C., Jr., Lee, J. C., Funes, J. G., et al. 2008, ApJS, 178, 247

Kennicutt, R. C., Jr., Hao, C.-N., Calzetti, D., et al. 2009, ApJ, 703, 1672

Kilborn, V. A., Forbes, D. A., Barnes, D. G., et al. 2009, MNRAS, 400, 1962

Kilborn, V. A., Koribalski, B. S., Forbes, D. A., Barnes, D. G., \& Musgrave,

R. C. 2005, MNRAS, 356, 77

Kourkchi, E., \& Tully, R. B. 2017, ApJ, 843, 16

Lee, J. C., Gil de Paz, A., Tremonti, C., et al. 2009, ApJ, 706, 599

Lewis, I., Balogh, M., De Propris, R., et al. 2002, MNRAS, 334, 673

Makarov, D., \& Karachentsev, I. 2011, MNRAS, 412, 2498

Malin, D. F., \& Carter, D. 1983, ApJ, 274, 534

Mamon, G. A. 1992, ApJ, 401, L3

Mancillas, B., Duc, P.-A., Combes, F., et al. 2019, A\&A, 632, A122

Marino, A., Bianchi, L., Rampazzo, R., Buson, L. M., \& Bettoni, D. 2010, A\&A,

511, A29

Marino, A., Rampazzo, R., Bianchi, L., et al. 2011a, MNRAS, 411, 311

Marino, A., Bianchi, L., Rampazzo, R., et al. 2011b, Ap\&SS, 335, 243 
Marino, A., Bianchi, L., Rampazzo, R., et al. 2011c, ApJ, 736, 154

Marino, A., Plana, H., Rampazzo, R., et al. 2013, MNRAS, 428, 476

Marino, A., Mazzei, P., Rampazzo, R., \& Bianchi, L. 2016, MNRAS, 459, 2212

Mazzei, P., Marino, A., Rampazzo, R., Galletta, G., \& Bettoni, D. 2014a, Adv. Space Res., 53, 950

Mazzei, P., Marino, A., \& Rampazzo, R. 2014b, ApJ, 782, 53

Mazzei, P., Rampazzo, R., Marino, A., et al. 2019, ApJ, 885, 165

Murugeshan, C., Kilborn, V., Obreschkow, D., et al. 2019, MNRAS, 483, 2398

Oh, S. H., Kim, W.-T., \& Lee, H. M. 2015, ApJ, 807, 73

Paccagnella, A., Vulcani, B., Poggianti, B. M., et al. 2016, ApJ, 816, L25

Panuzzo, P., Vega, O., Bressan, A., et al. 2007, ApJ, 656, 206

Panuzzo, P., Rampazzo, R., Bressan, A., et al. 2011, A\&A, 528, A10

Parkash, V., Brown, M. J. I., Jarrett, T. H., Fraser-McKelvie, A., \& Cluver, M. E. 2019, MNRAS, 485, 3169

Pearson, S., Besla, G., Putman, M. E., et al. 2016, MNRAS, 459, 1827

Phillips, M. M., Jenkins, C. R., Dopita, M. A., Sadler, E. M., \& Binette, L. 1986, AJ, 91, 1062

Poggianti, B. M., von der Linden, A., De Lucia, G., et al. 2006, ApJ, 642, 188 Ramella, M., Geller, M. J., Pisani, A., \& da Costa, L. N. 2002, AJ, 123, 2976 Rampazzo, R. 1988, A\&A, 204, 81

Rampazzo, R., Plana, H., Longhetti, M., et al. 2003, MNRAS, 343, 819

Rampazzo, R., Panuzzo, P., Vega, O., et al. 2013, MNRAS, 432, 374

Rampazzo, R., Mazzei, P., Marino, A., et al. 2017, A\&A, 602, A97

Rampazzo, R., Mazzei, P., Marino, A., et al. 2018, Ap\&SS, 363, 80
Rampazzo, R., Omizzolo, A., Uslenghi, M., et al. 2020, A\&A, 640, A38 Ribeiro, A. L. B., de Carvalho, R. R., Capelato, H. V., \& Zepf, S. E. 1998, ApJ, 497, 72

Roming, P. W. A., Kennedy, T. E., Mason, K. O., et al. 2005, Space Sci. Rev., 120,95

Rossa, J., \& Dettmar, R. J. 2003, A\&A, 406, 505

Roy, J.-R., \& Walsh, J. R. 1986, MNRAS, 223, 39

Ryan-Weber, E. V., Webster, R. L., \& Staveley-Smith, L. 2003, MNRAS, 343, 1195

Schawinski, K., Kaviraj, S., Khochfar, S., et al. 2007, ApJS, 173, 512

Serra, P., Oosterloo, T., Morganti, R., et al. 2012, MNRAS, 422, 1835

Tago, E., Einasto, J., Saar, E., et al. 2008, A\&A, 479, 927

Tal, T., van Dokkum, P. G., Nelan, J., \& Bezanson, R. 2009, AJ, 138, 1417

Tandon, S. N., Subramaniam, A., Girish, V., et al. 2017, AJ, 154, 128

Tody, D. 1986, in The IRAF Data Reduction and Analysis System, ed. D. L. Crawford, SPIE Conf. Ser., 627, 733

Toomre, A., \& Toomre, J. 1972, ApJ, 178, 623

Trinchieri, G., Noris, L., \& di Serego Alighieri, S. 1997, A\&A, 326, 565

Tully, R. B. 1988, Nearby Galaxies Catalog

Tully, R. B. 2015, AJ, 149, 54

Tully, R. B., \& Fisher, J. R. 1988, Catalog of Nearby Galaxies

Vega, O., Bressan, A., Panuzzo, P., et al. 2010, ApJ, 721, 1090

Vulcani, B., Poggianti, B. M., Finn, R. A., et al. 2010, ApJ, 710, L1

Weil, M. L., \& Hernquist, L. 1993, ApJ, 405, 142

Werk, J. K., Putman, M. E., Meurer, G. R., et al. 2010, AJ, 139, 279 


\section{Appendix A: Dorado members}

The Dorado group is indicated as PGC1 14765, that is with the PGC (Principal Galaxy Catalogue) number of the main galaxy NGC 1553 in Kourkchi \& Tully (2017) and counts 31 members. They are indicated with the number $\mathrm{N}$, reported in column 1 of Table A.1. From this list we removed 2MASXJ041059835628496, which corresponds to NGC 1536, which is already present in the list, reducing the number of members to 30 . In Table A.1 we add PGC 75125 from the Firth et al. (2006) Dorado sample to the re-defined Kourkchi \& Tully (2017) list. PGC 75125 is in the same frame as IC 2058 (see Table 1 and Fig. 1) and is a physical companion of this galaxy.

From the Kourkchi \& Tully (2017) paper, the table reports: member number (column 1);

Right Ascension (column 2);

Declination (column 3);

PGC number (column 4);

Galaxy name (column 5);

Morphological Type (column 6);

the total apparent $B$-band magnitude (column 7);

the total apparent $K_{s}$-band magnitude (column 8);

the logarithm of the total apparent $K_{s}$-band magnitude in solar luminosity (column 9);

the heliocentric recession velocity (column 10); 11).

the galaxy distance in Mpc and the percentage error (column

The distribution of the morphological type and of the heliocentric velocity are shown in Fig. A.1.
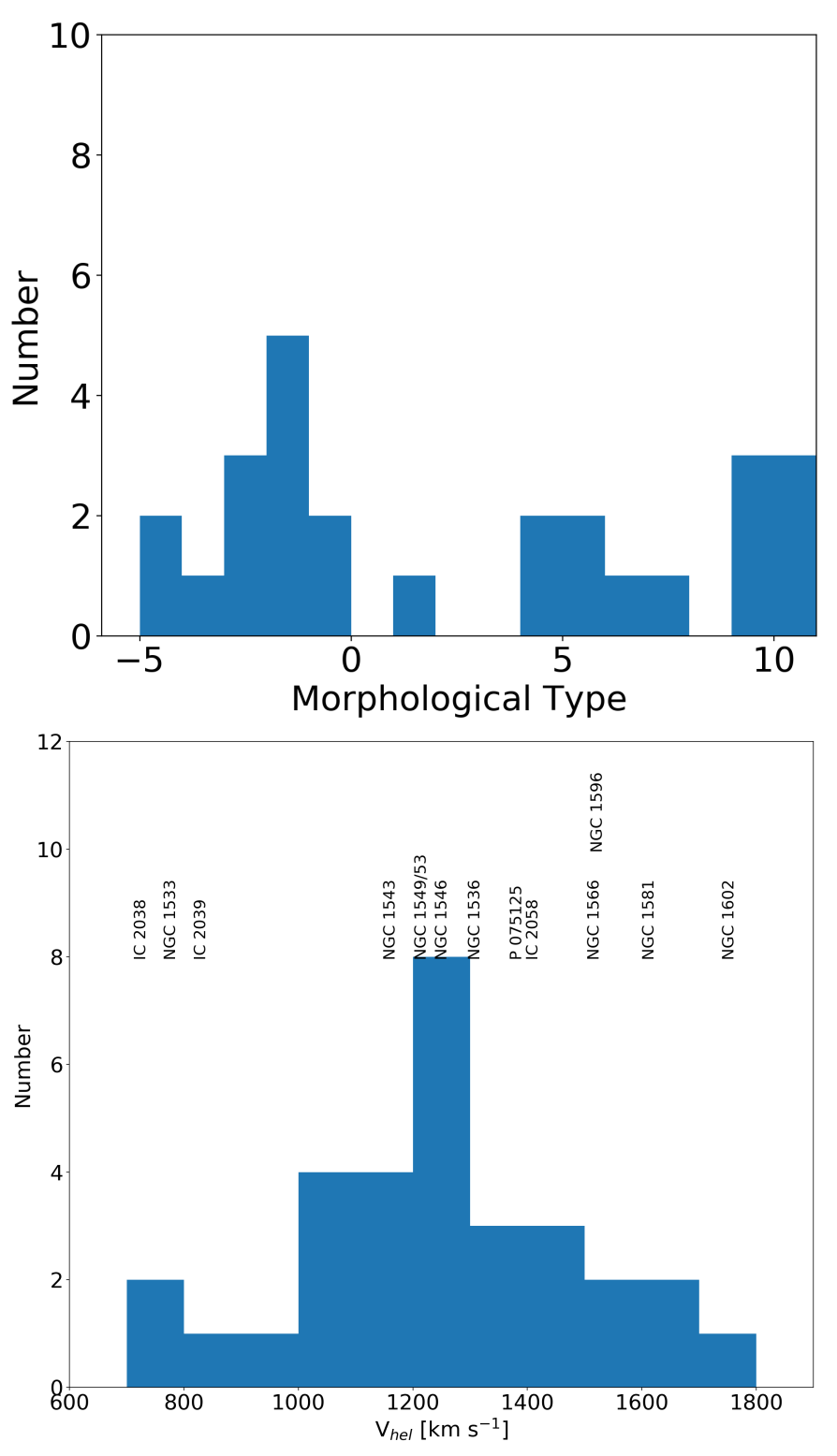

Fig. A.1. From top to bottom, morphological type (only 26/31 objects in Table A. 1 have a morphological classification) and heliocentric velocity distribution of Dorado members in Table A.1. Members included in this study are indicated in correspondence with their heliocentric velocity. 


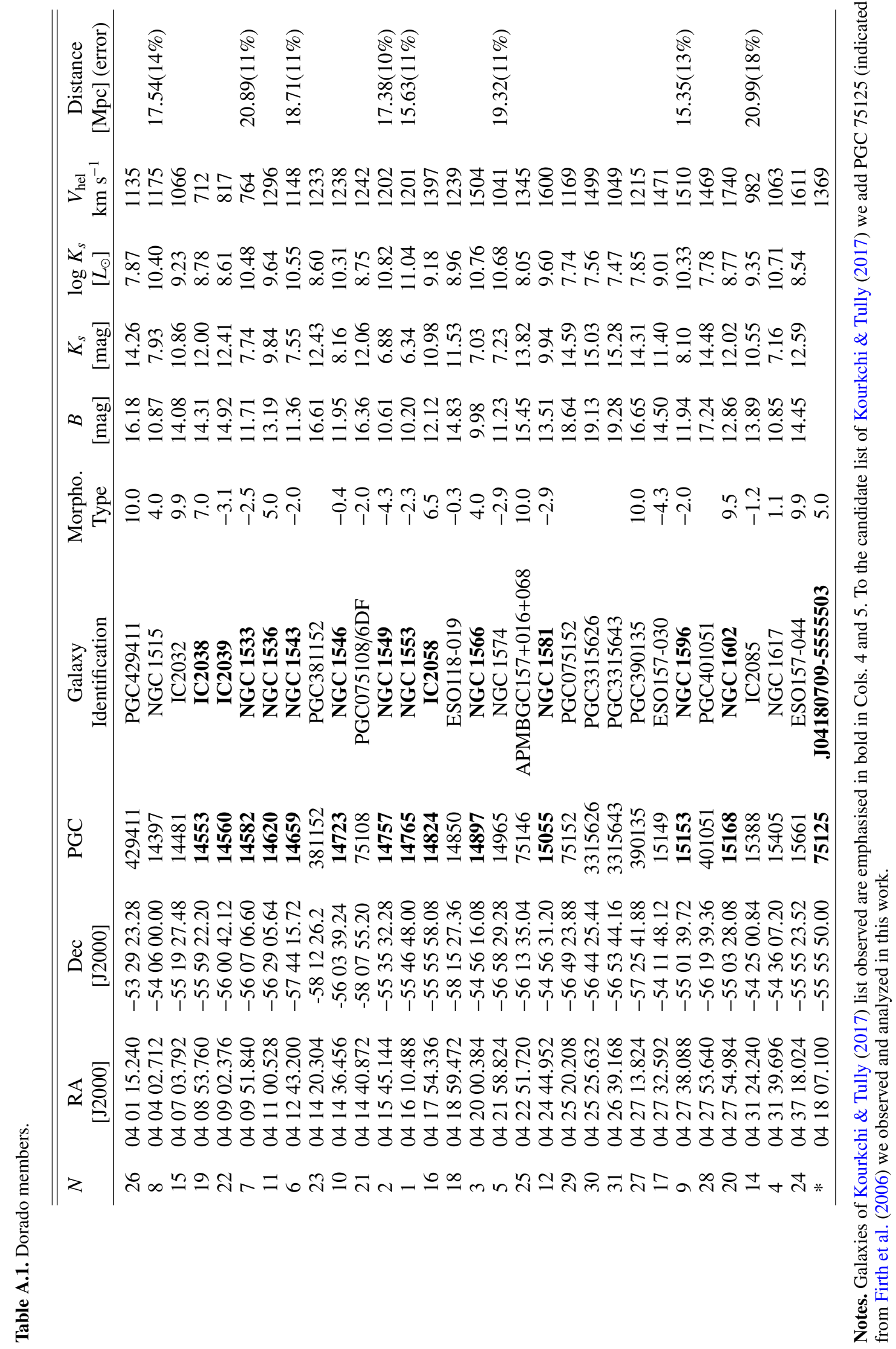




\section{Appendix B: Comparison with the literature}

Only one galaxy, NGC 1566, in our sample has been previously observed in the same band. Hoopes et al. (2001) and Kennicutt et al. (2009) performed $\mathrm{H} \alpha$ and $\mathrm{H} \alpha+[\mathrm{N} \mathrm{II}]$ imaging of the entire galaxy, respectively. Hoopes et al. (2001) observed at the CTIO 0.9m telescope using $\mathrm{H} \alpha 6602 / 20$ filter and an $R$ band filter to remove the stellar continuum. The 6602/20 filter, centred at $\lambda=6596 \AA$ has a $F W H M=18 \AA$ and a transmission of $70 \%$ at the central wavelength. As a consequence, the [NII] emission does not contaminate the measure. The paper reports the $\mathrm{H} \alpha$ luminosity $L_{\mathrm{H} \alpha}=31.65 \pm 0.6 \times 10^{33} \mathrm{~W}$ (adopting a distance of $17.49 \mathrm{Mpc}$ ). This is equivalent to a flux of $880 \times 10^{-14} \mathrm{erg} \mathrm{s}^{-1} \mathrm{~cm}^{-2}$.

In their Table 1, Kennicutt et al. (2009) reported a measure of $\log (\mathrm{H} \alpha+[\mathrm{N} \mathrm{II}])=-10.9 \pm 0.1 \mathrm{erg} \mathrm{s}^{-1} \mathrm{~cm}^{-2}$ corresponding to a $\mathrm{H} \alpha+[\mathrm{N} \mathrm{II}]$ flux of $1318 \times 10^{-14} \mathrm{erg} \mathrm{s}^{-1} \mathrm{~cm}^{-2}$.

Kennicutt et al. (2009) adopted the $[\mathrm{NII}] / \mathrm{H} \alpha=0.62 \pm 0.06$ ratio to correct for the [NII] emission obtained by Hawley \& Phillips (1980) from the study of three H II regions. Roy \& Walsh (1986) performed long-slit scanning spectroscopy at low resolution $(\Delta \lambda=0.7 \AA)$ covering a rectangular region of $120^{\prime \prime} \times 16^{\prime \prime}$ positioned on the NW spiral arm of NGC 1566 . They measured the lines fluxes in five H II regions. From their work we derived the average ratio of $[\mathrm{NII}] / \mathrm{H} \alpha=0.38$, which is significantly reduced with respect to that found by Hawley \& Phillips (1980).

Assuming as the net $\mathrm{H} \alpha$ flux the value of Hoopes et al. (2001), the $\mathrm{H} \alpha+\left[\mathrm{N} \mathrm{II]} \mathrm{flux} \mathrm{is} 1425.6 \pm 50.2 \times 10^{-14} \mathrm{erg} \mathrm{s}^{-1}\right.$ $\mathrm{cm}^{-2}$ and $1214.4 \pm 33.4 \times 10^{-14} \mathrm{erg} \mathrm{s}^{-1} \mathrm{~cm}^{-2}$ adopting the Hawley \& Phillips (1980) and Roy \& Walsh (1986) [NII]/H $\alpha$ ratios, respectively.

We conclude that our value $1246.7 \pm 125.1 \times 10^{-14} \mathrm{erg} \mathrm{s}^{-1}$ $\mathrm{cm}^{-2}$ (Table 2) agrees, within errors, with both the Hoopes et al. (2001) $\mathrm{H} \alpha$ estimate, once adopted the Roy \& Walsh (1986) $[\mathrm{NII}] / \mathrm{H} \alpha$ ratio, and with the $\mathrm{H} \alpha+[\mathrm{NII}]$ measure by Kennicutt et al. (2009).

\section{Appendix C: Images in the continuum and in the $\mathrm{H} \alpha+[\mathrm{N} \mathrm{II}]$ filter}

We present here the images of the galaxies observed in $\mathrm{H} \alpha+\left[\begin{array}{ll}\mathrm{N} & \mathrm{II}\end{array}\right]$. For galaxies larger than the du Pont FOV $(8.85 \times 8$ 85) we obtained a mosaic of images. Left and right panels show continuum and $\mathrm{H} \alpha$ images, respectively. Stellar residuals, sometimes present in the $\mathrm{H} \alpha$ images due to bright stars, were removed. 
R. Rampazzo et al.: $\mathrm{H} \alpha$ imaging of the Dorado group backbone

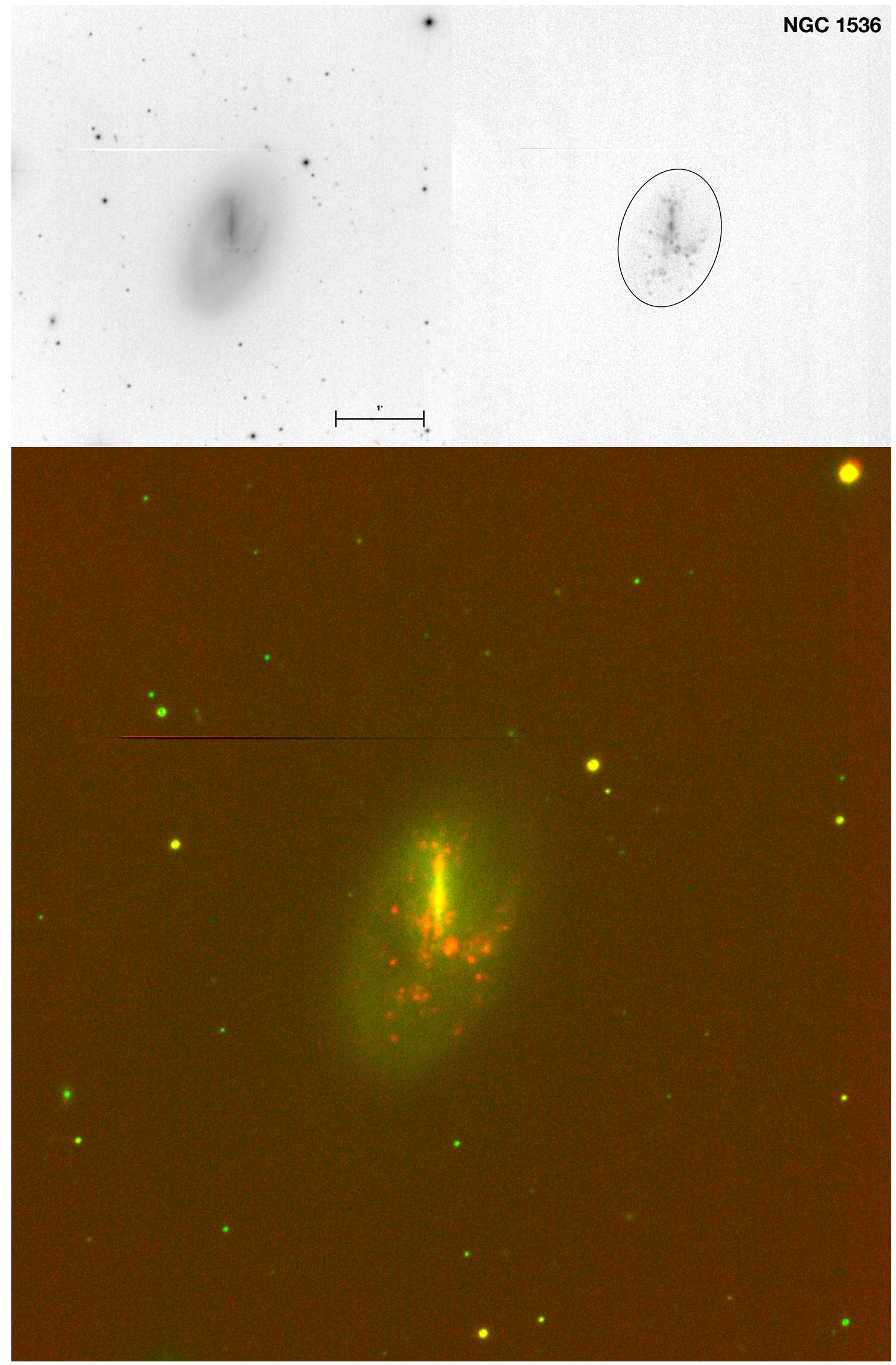

Fig. C.1. As in Fig. 3 but for NGC 1536. The image size is $5^{\prime} \times 5^{\prime}$. 
A\&A 643, A176 (2020)

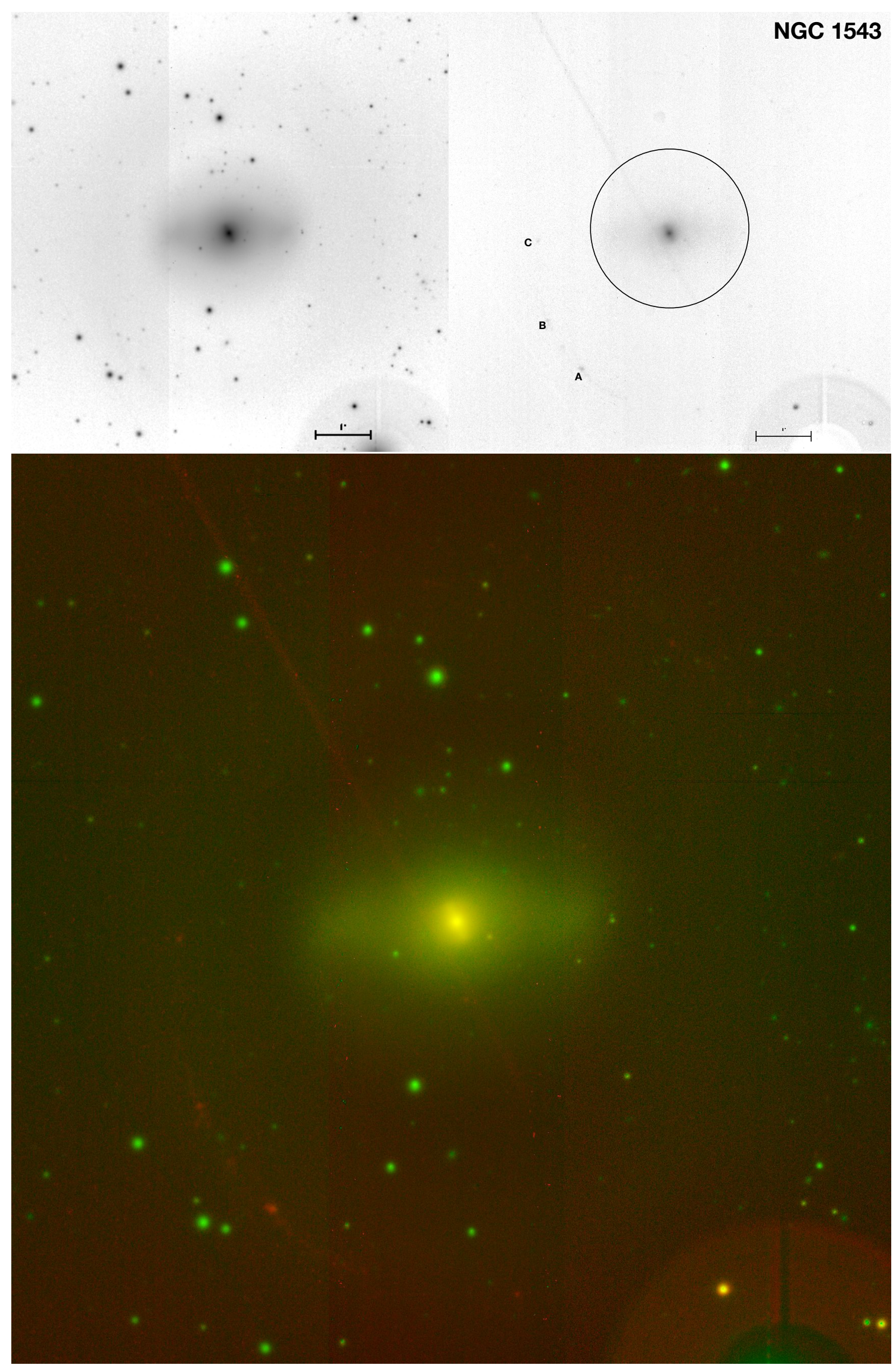

Fig. C.2. As in Fig. 3 but for NGC 1543. Labels A, B, and C (top right panel) indicate emission areas along the outer ring of the galaxy. The total flux in the ring is $3.72 \pm 0.37 \times 10^{-14} \mathrm{erg} \mathrm{s}^{-1} \mathrm{~cm}^{-2}$. The image size is $8^{\prime} \times 8^{\prime}$. 
R. Rampazzo et al.: $\mathrm{H} \alpha$ imaging of the Dorado group backbone

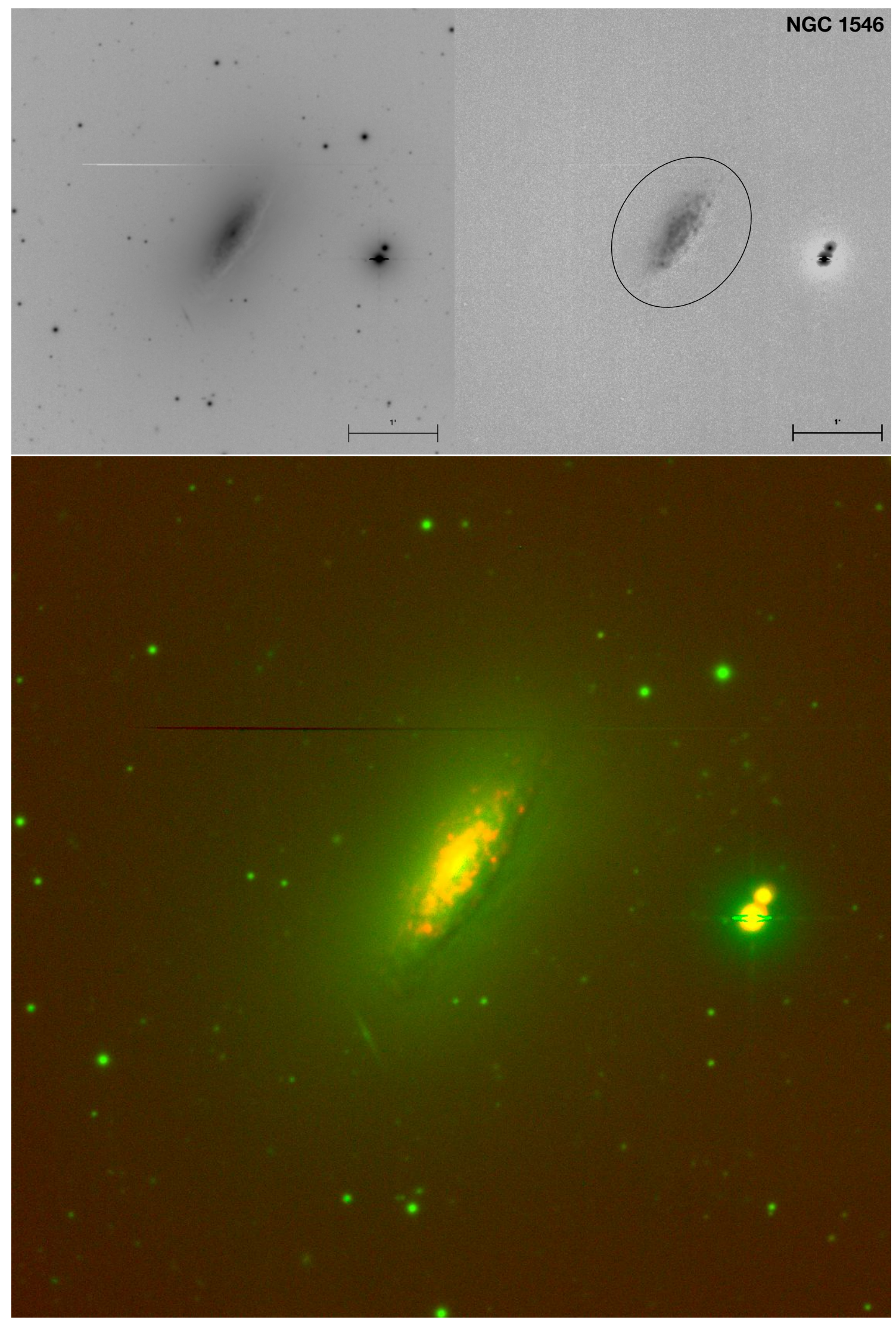

Fig. C.3. As in Fig. 3 but for NGC 1546. The image size is $5^{\prime} \times 5^{\prime}$. 
A\&A 643, A176 (2020)

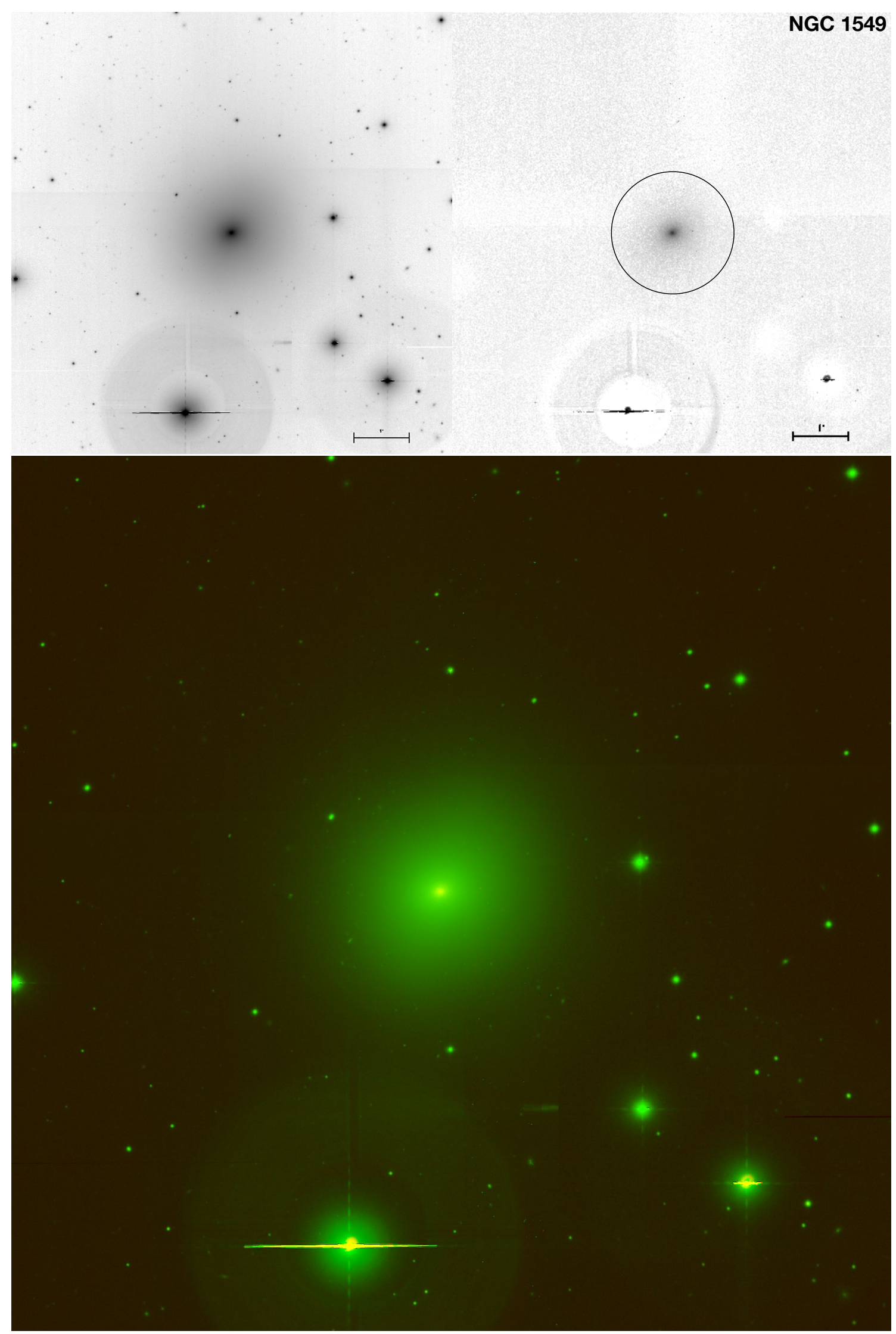

Fig. C.4. As in Fig. 3 but for NGC 1549 The image size is $8^{\prime} \times 8^{\prime}$. 
R. Rampazzo et al.: $\mathrm{H} \alpha$ imaging of the Dorado group backbone

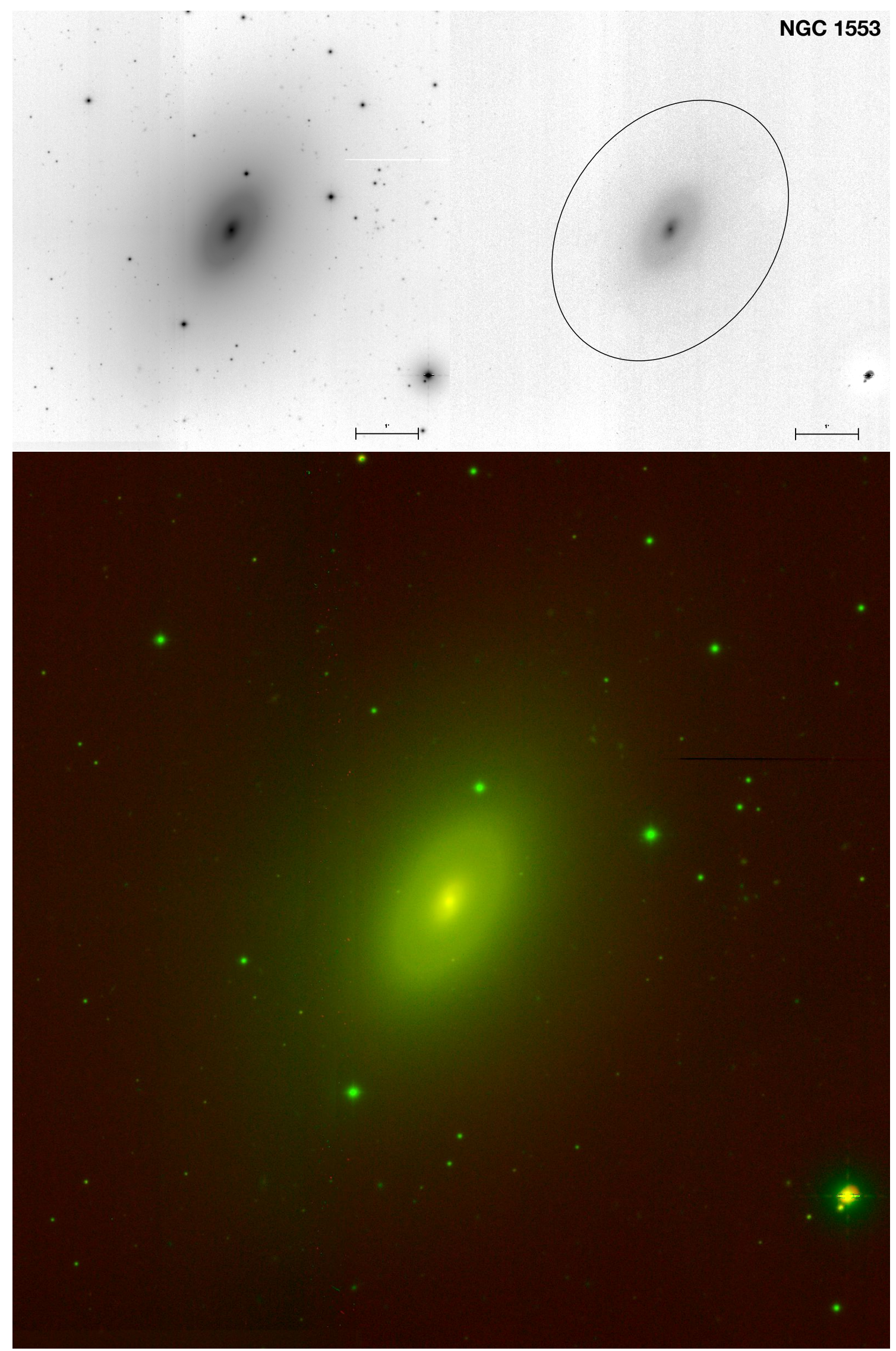

Fig. C.5. As in Fig. 3 but for NGC 1553. The image size is $7^{\prime} \times 7^{\prime}$. Two $\mathrm{H} \alpha$-bright regions are shown in detail in Fig. 9. 
A\&A 643, A176 (2020)

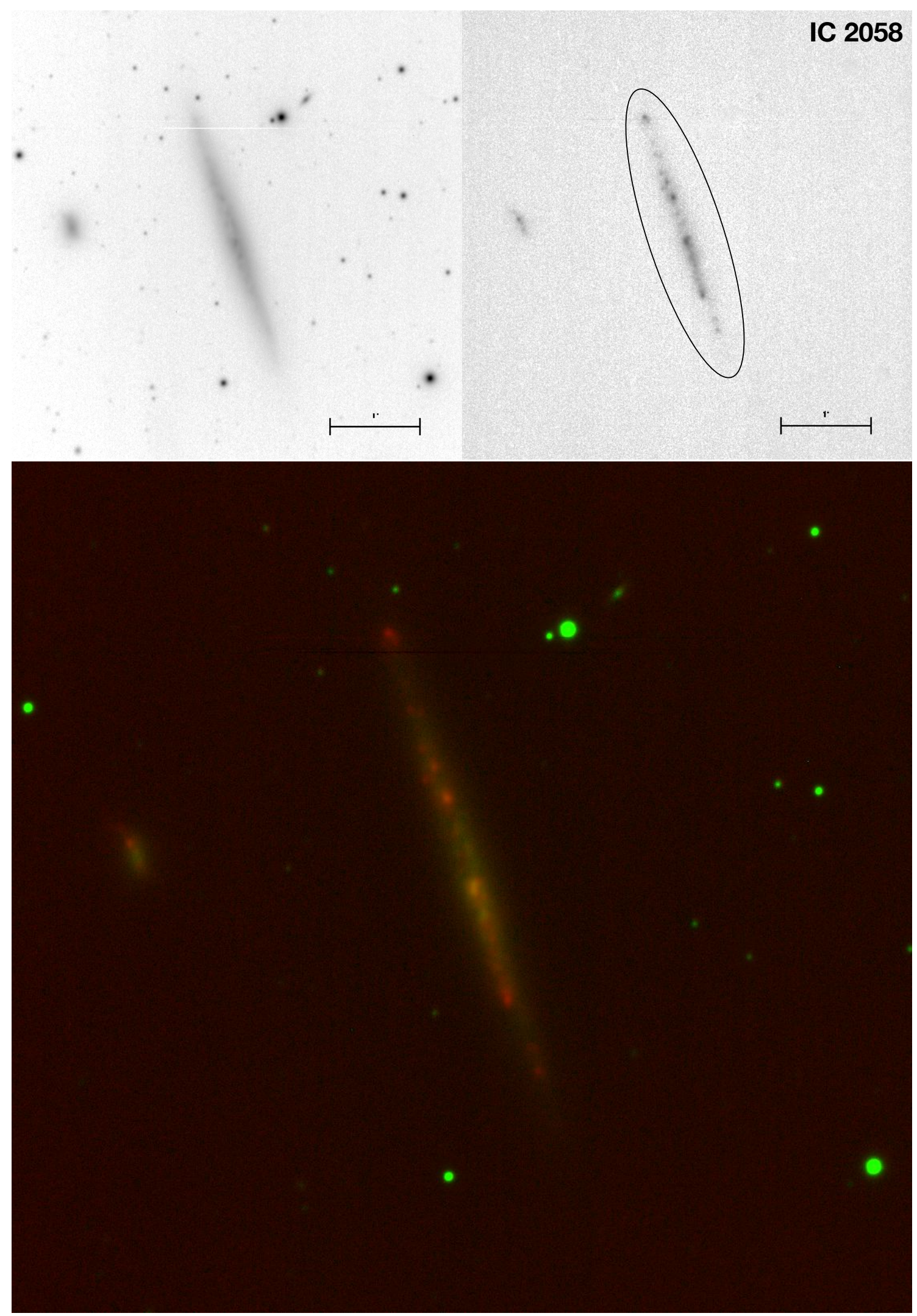

Fig. C.6. As in Fig. 3 but for IC 2058. The image size is $5^{\prime} \times 5^{\prime}$. The physical companion of IC 2058, PGC 75125, is visible east of the galaxy. 
R. Rampazzo et al.: H $\alpha$ imaging of the Dorado group backbone

PGC 075125
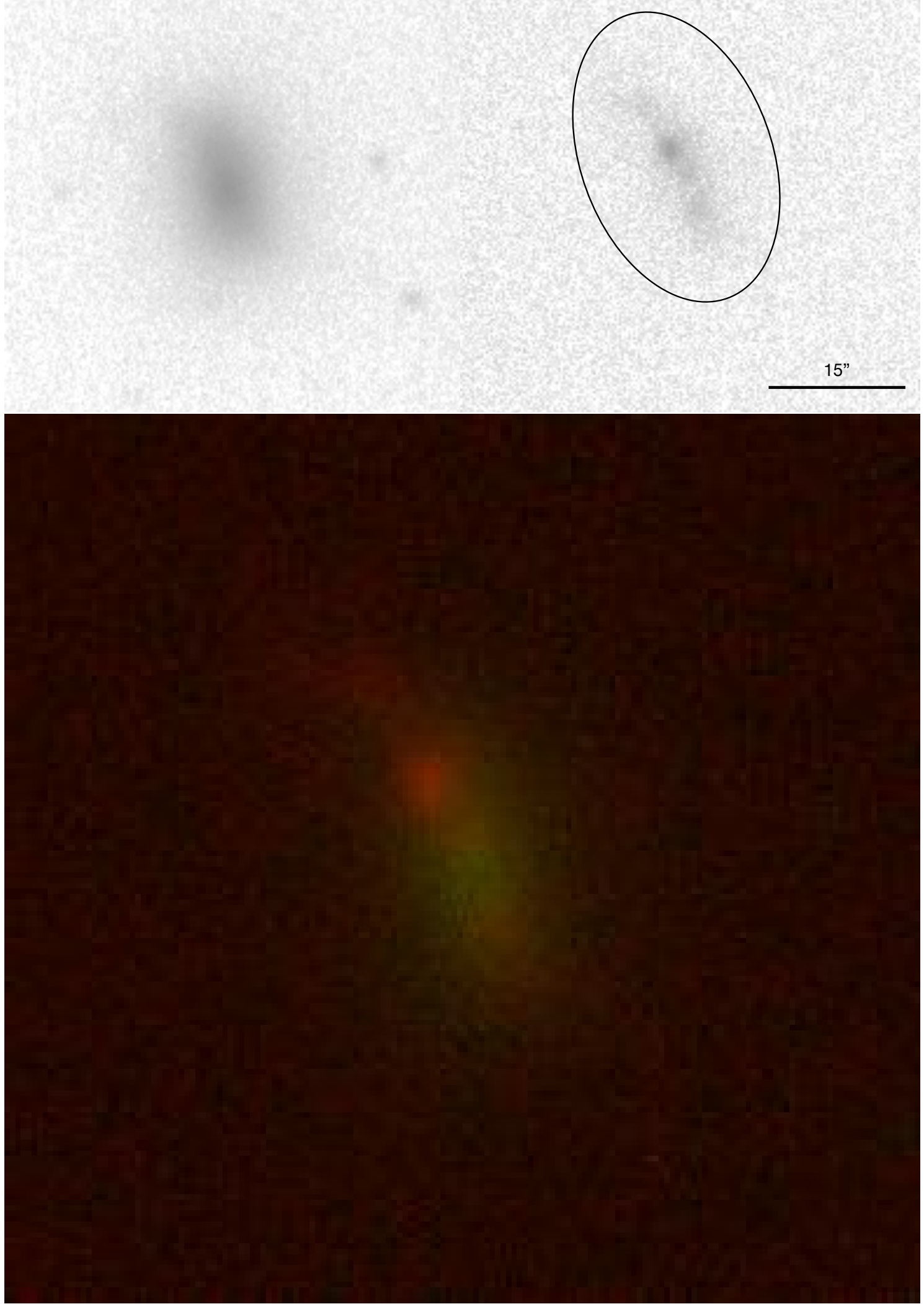

Fig. C.7. As in Fig. 3 but for PGC 75125. The image size is $50^{\prime \prime} \times 50^{\prime \prime}$. The galaxy is classified as spiral $(T=5 \pm 3)$ in HyperLeda. In the continuum the galaxy structure appears boxy and without spiral arms. The $\mathrm{H} \alpha+[\mathrm{NII}]$ image shows a warped, clumpy star forming lane crossing the galaxy. 
A\&A 643, A176 (2020)

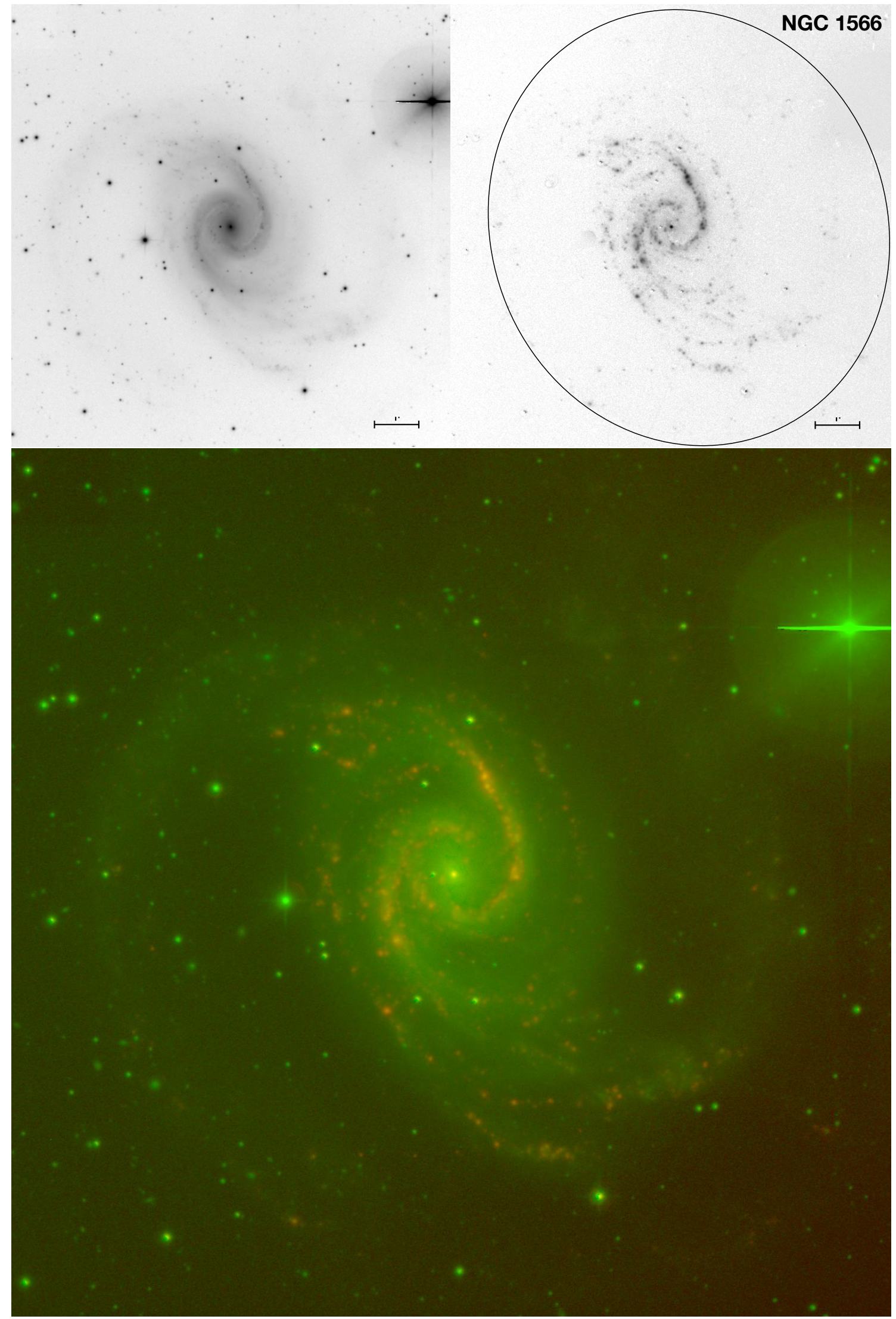

Fig. C.8. As in Fig. 3 but for NGC 1566. The image size is $10^{\prime} \times 10^{\prime}$. 
R. Rampazzo et al.: $\mathrm{H} \alpha$ imaging of the Dorado group backbone

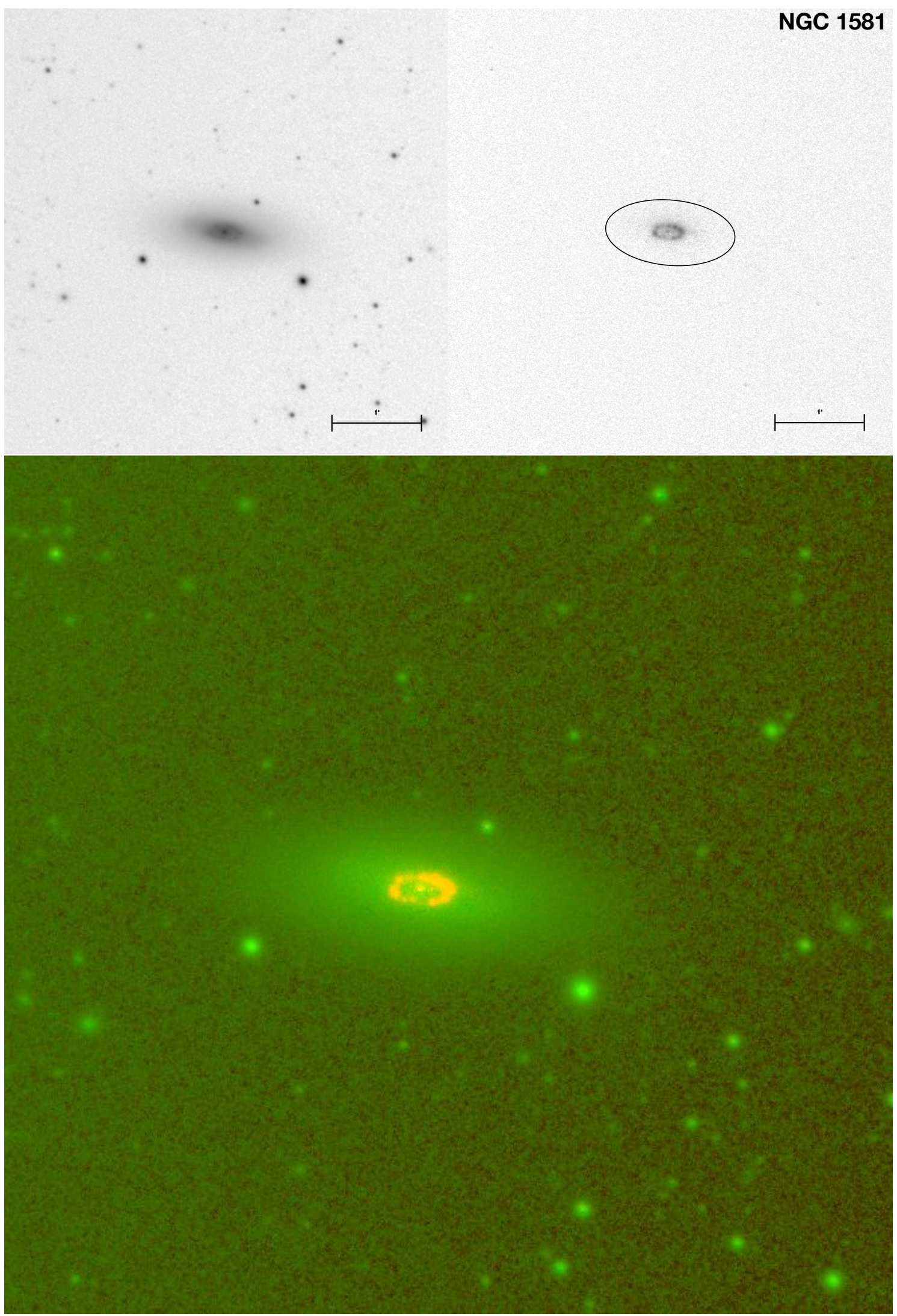

Fig. C.9. As in Fig. 3 but for NGC 1581. The image size is $5^{\prime} \times 5^{\prime}$. 


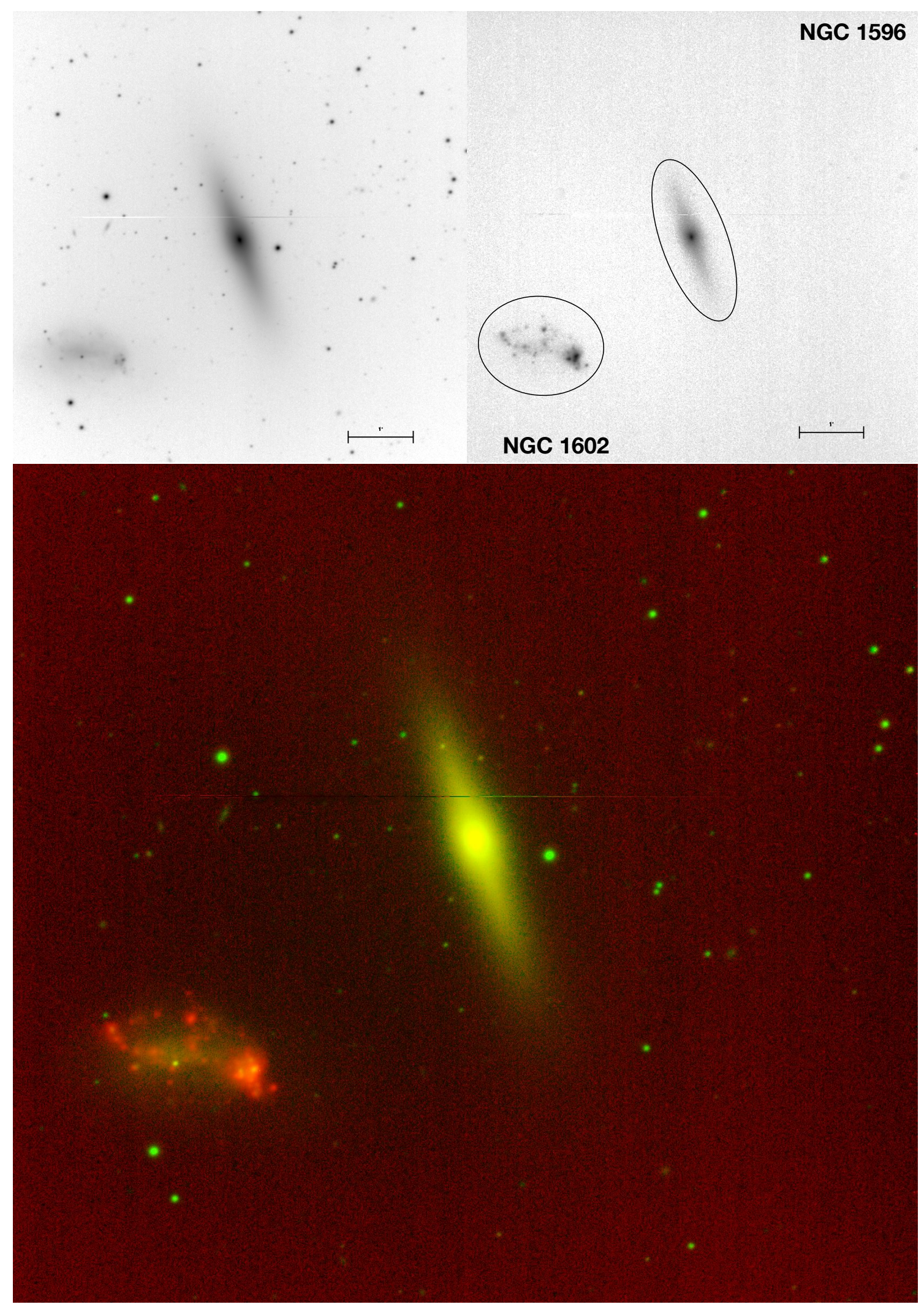

Fig. C.10. As in Fig. 3 but for the physical pair (Bureau \& Chung 2006) formed by NGC 1596 (NW) and NGC 1602 (SE). The image size is 7’ $\times 7^{\prime}$. 